Portland State University

PDXScholar

\title{
Quantitative Assessment of the Lewis Acidity of Diaryliodonium Salts
}

Florian H. Guillot

Portland State University

Follow this and additional works at: https://pdxscholar.library.pdx.edu/honorstheses

Let us know how access to this document benefits you.

\section{Recommended Citation}

Guillot, Florian H., "Quantitative Assessment of the Lewis Acidity of Diaryliodonium Salts" (2018).

University Honors Theses. Paper 573.

https://doi.org/10.15760/honors.580

This Thesis is brought to you for free and open access. It has been accepted for inclusion in University Honors Theses by an authorized administrator of PDXScholar. Please contact us if we can make this document more accessible: pdxscholar@pdx.edu. 
Quantitative Assessment of the Lewis Acidity of Diaryliodonium Salts

\section{by}

\section{Florian Guillot}
An undergraduate honors thesis submitted in partial fulfillment of the requirements for the degree of
Bachelor of Science
in
University Honors
and
Mathematics

Thesis Adviser

David R. Stuart

Portland State University 


\section{Abstract}

Diaryliodonium salts are promising novel reagents in arylation chemistry as they represent an efficient, inexpensive and non-toxic alternative to the most commonly used heavy-metal-based oxidants and organometallic catalysts. While the electrophilicity of these reagents has been widely accepted, few studies have made use of these new reagents as Lewis acids. In order to further the understanding of diaryliodonium salts, this work aims to quantify the Lewis acidity of a variety of $\operatorname{aryl}(2,4,6$-trimethoxyphenyl)iodonium salts. To this end, the Guttmann-Beckett method was used in which NMR titrations of triethylphosphine oxide (TPO) and diaryliodonium salts were performed in deuterated acetonitrile. Nonlinear regression was then used to determine the association constants of each compound. The effect of counter anion identity was studied in phenyl(2,4,6-trimethoxyphenyl)iodonium salts and resulted in the following ranking, from lowest to highest acidity, based on counter anions: TFA $<\mathrm{PF}_{6}<\mathrm{OTf}<\mathrm{BF}_{4}$. Lastly, para- $t \mathrm{Bu}-$ phenyl(2,4,6-trimethoxyphenyl)iodonium tosylate yielded the lowest Lewis acidity measurement amongst all diaryliodonium salts studied. 


\section{Acknowledgments}

First of all, I would like to express my sincere gratitude to my adviser, Dr. Stuart, for welcoming me into his laboratory and taking the time and effort necessary to guide me through this project. I am thankful for Dr. Stuart's continuous support, both in and out of the lab, and his dedication to facilitating students' growth. I will always value Dr. Stuart's strong work ethic and academic expertise. I am grateful for the trust placed in me and for the independence I was given throughout this study.

This work would not have been possible without the support from all the graduate students in the Stuart Group, who always enthusiastically shared their expertise with me. Particular thanks go to Thomas for recruiting me, for introducing me to the world of organic chemistry research and for giving me the will and courage to dive in. I am also extremely grateful to have worked with Souradeep, whom I thank for his patience and diligence in guiding me through each experiment, and for generously sharing his unpublished data with me. I cannot thank Sasha enough for sharing her hood, knowledge and equipment, and for always conveying encouragement and positivity in the lab. I thank Rory for his help, his wonderful humor and broad knowledge.

Finally, I would like to thank my family and friends, who supported me through my studies and constantly gave me inspiration to keep learning. 


\section{Table of Contents}

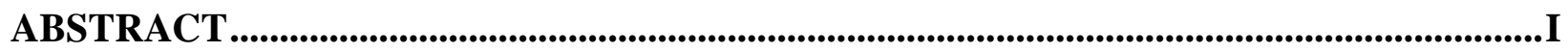

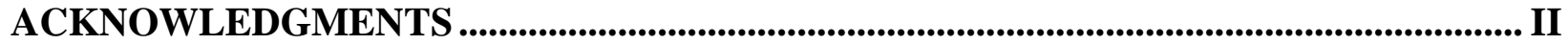

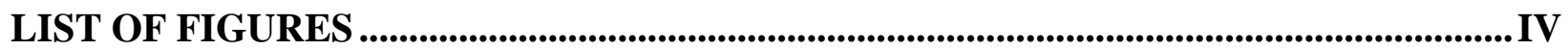

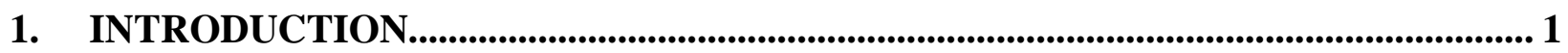

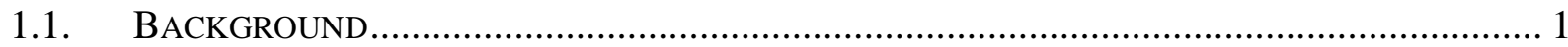

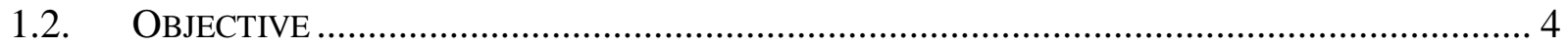

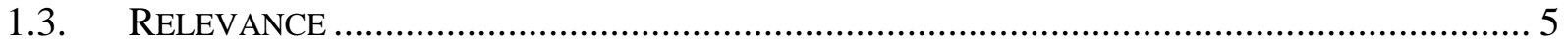

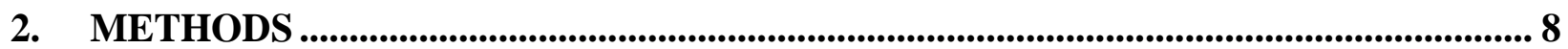

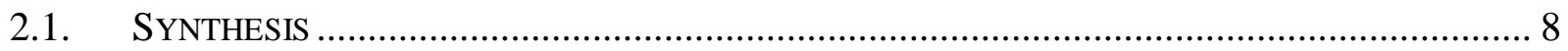

2.2. GUTMANN-BECKETT METHOD........................................................................... 11

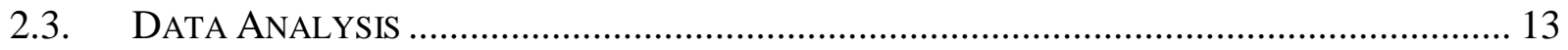

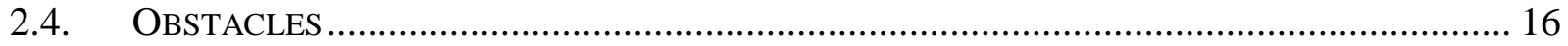

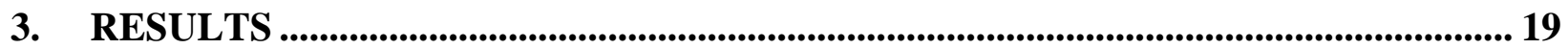

3.1. PhenYL(TMP)IOdONIUM HEXAFLUOROPHOSPhate ..................................................... 19

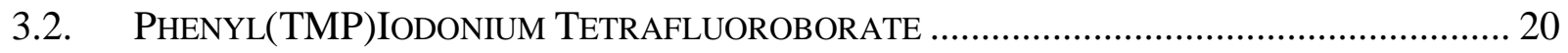

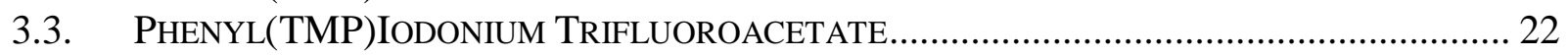

3.4. PHENYL(TMP)IODONIUM TRIFLATE ..................................................................... 23

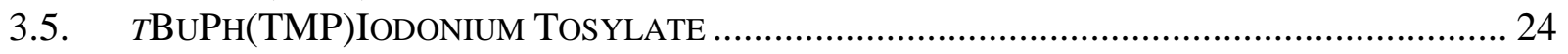

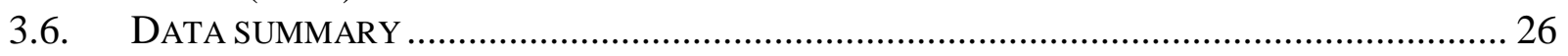

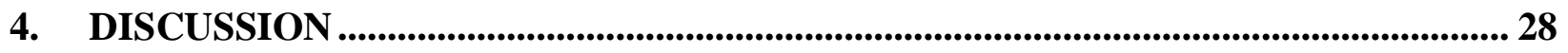

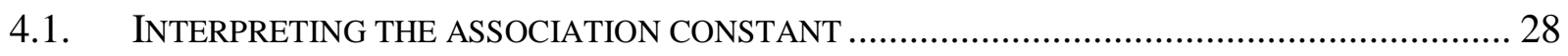

4.2. OBSERVED TREND IN ARYL(TMP)IODONIUM SALTS................................................... 28

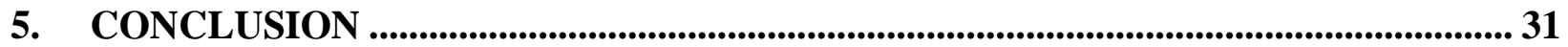

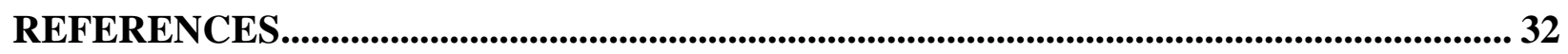

APPENDICES .................................................................................................................................................. 34

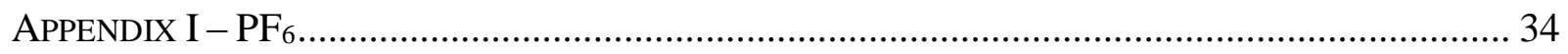

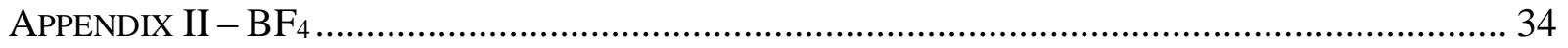

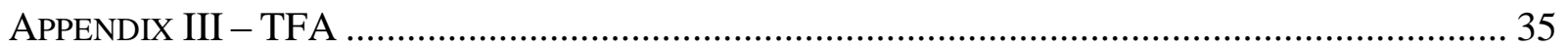

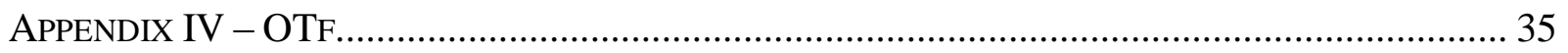

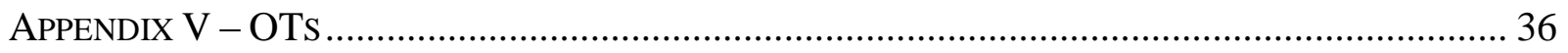




\section{List of Figures}

Figure 1. Frequency of "diaryliodonium salt" in the chemical literature ...................................... 1

Figure 2. General pseudotrigonal bipyramid structure of diaryliodonium salts and molecular orbital of three-center-four-electron bond ............................................................... 2

Figure 3. Crystal structure of diphenyliodonium hexafluorophosphate ….................................. 3

Figure 4. Synthesis of aryl(2,4,6-trimethoxyphenyl)iodonium tosylate ...................................... 8

Figure 5. Counter anion exchange for synthesis of aryl(TMP) iodonium salts ............................ 9

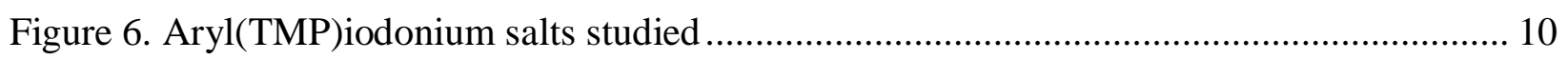

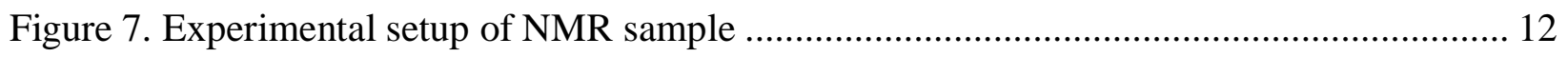

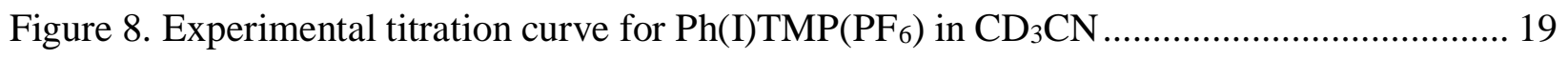

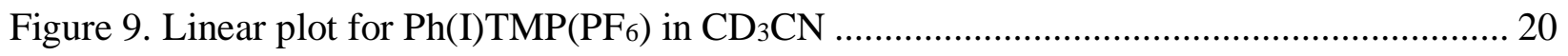

Figure 10. Experimental titration curve for $\mathrm{Ph}(\mathrm{I}) \mathrm{TMP}\left(\mathrm{BF}_{4}\right)$ in $\mathrm{CD}_{3} \mathrm{CN}$................................. 21

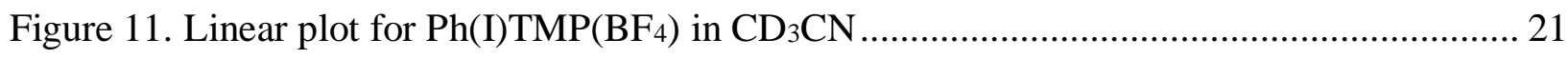

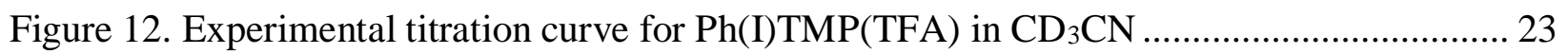

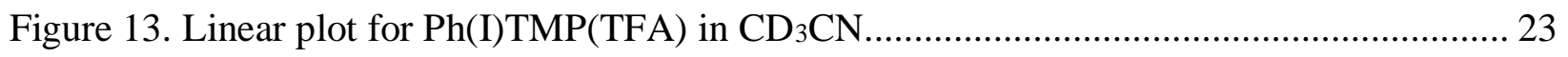

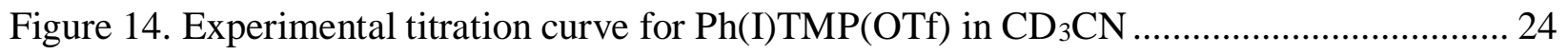

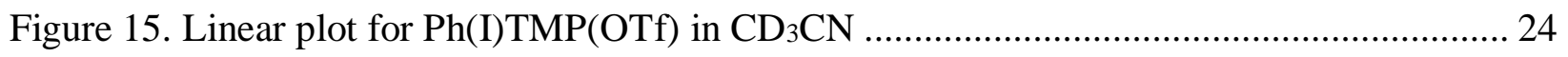

Figure 16. Experimental titration curve for $t \mathrm{BuPh}(\mathrm{I}) \mathrm{TMP}(\mathrm{OTs})$ in $\mathrm{CD}_{3} \mathrm{CN}$.............................. 25

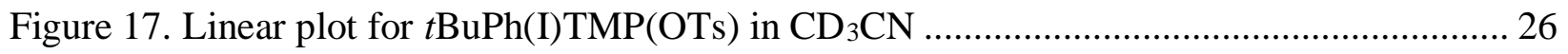

Figure 18. Summary of titration curves for different counter anions ........................................ 27

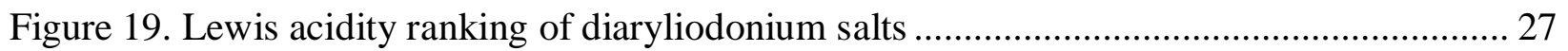




\section{Introduction}

\subsection{Background}

Although first synthesized as early as 1894 by Hartmann and Meyer $^{1}$, diaryliodonium salts have long been overlooked by the organic chemistry synthesis community. ${ }^{2}$ The general neglect for those iodonium compounds was mainly due to the time-consuming and inefficient nature of their synthesis. ${ }^{2}$ For several decades, the research community focused primarily on more readily available alternatives such as heavy-metal-based oxidants and expensive organometallic catalysts. ${ }^{2}$

Currently, diaryliodonium salts generate significant interest in the research community as new synthetic methods and novel applications emerge (Figure 1). ${ }^{3}$ Diaryliodonium salts in particular found acclaim in arylation chemistry thanks to their mild and selective reactivity combined with their stable, inexpensive and non-toxic nature. ${ }^{2}$

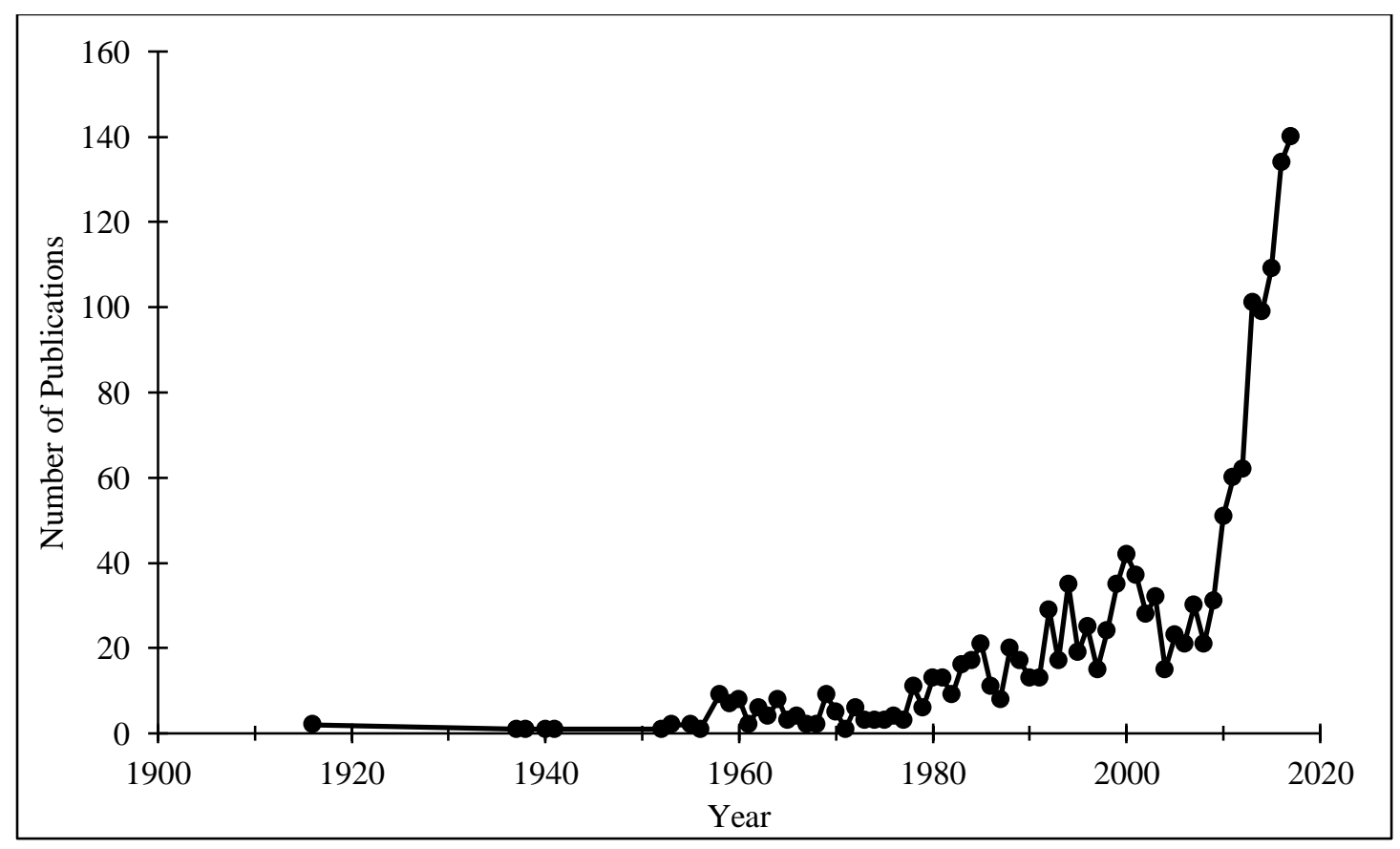

Figure 1. Frequency of "diaryliodonium salt" in the chemical literature 
As the heaviest of the stable halogens, iodine is often classified as a nonmetal. However, iodine-bearing compounds often exhibit unusual intermolecular bonding due to the large size of the iodine atom. ${ }^{4}$ Hypervalent iodine compounds, in particular, contain a hypervalent bond that originates from the overlap of iodine's $5 p$ orbital with the orbitals on the two ligands. This linear three-center-four-electron bond (Figure 2) is highly polar, and longer and weaker than a traditional covalent bond. ${ }^{4,5}$ Diaryliodonium salts are a specific subset of iodine compounds of the form [Ar$\mathrm{I}-\mathrm{Ar}]^{+} \mathrm{X}^{-}$(Figure 2). They are thus classified as hypervalent compounds, and more precisely $\lambda^{3}-$ compounds according to IUPAC (International Union of Pure and Applied Chemistry) nomenclature. 6
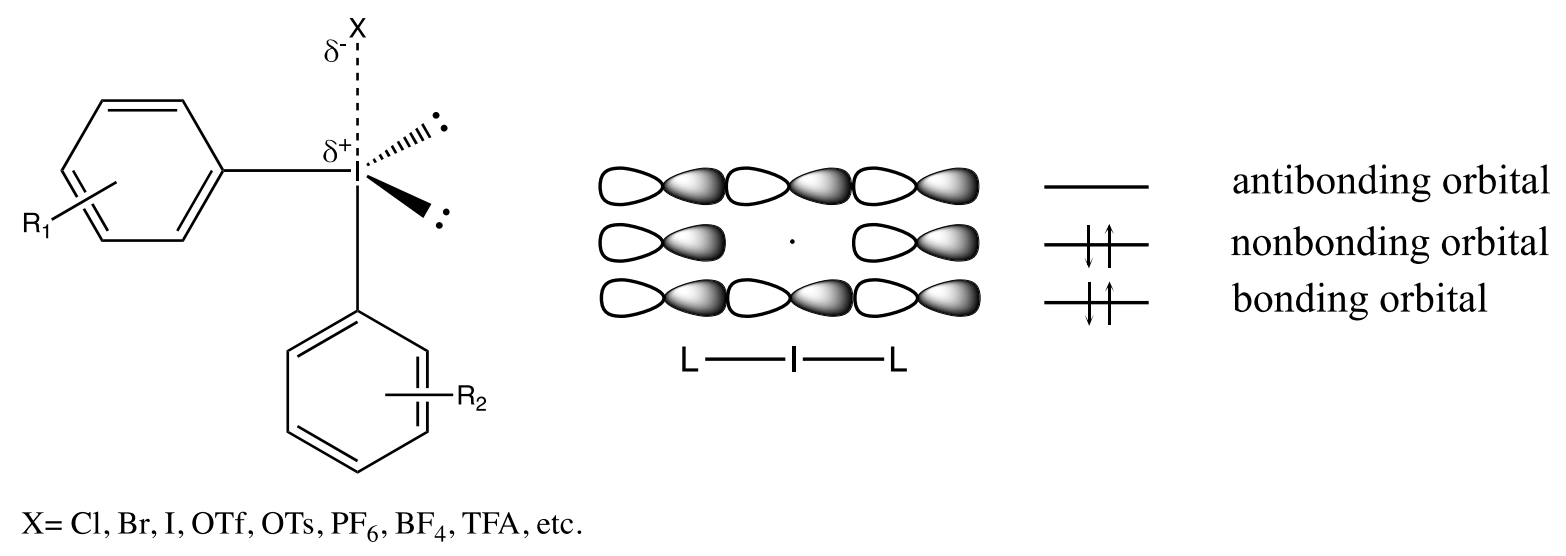

Figure 2. General pseudotrigonal bipyramid structure of diaryliodonium salts and

\section{molecular orbital of three-center-four-electron bond ${ }^{5}$}

Although the concept of hypervalency has been sharply criticized by theoretical chemists, ${ }^{7,8}$ the term "hypervalent" has been overwhelmingly accepted by synthetic chemists. Instead of the regular octet of electrons surrounding the iodine center, hypervalent iodine compounds contain ten or more electrons in their valence shells.

Due to this hypervalent bond and the presence of a node in its nonbonding orbital, diaryliodonium salts are highly electron-deficient at the iodine center. ${ }^{2,9}$ This property confers 
excellent reactivity of those salts and allows them to easily react with various nucleophiles. In addition to their highly electron-deficient nature, diaryliodonium salts also benefit from the excellent leaving-group of the Ar-I moiety. The leaving ability of Ph-I, for example, has been found to be about $10^{6}$ times greater than the triflate anion in the corresponding iodonium salt. ${ }^{10}$

Structurally, crystallized diaryliodonium salts have been found to have a T-shaped geometry with an Ar-I-Ar angle close to $90^{\circ}$, as illustrated by the crystal structure of diphenyliodonium hexafluorophosphate $\left(\mathrm{Ph}_{2} \mathrm{I}-\mathrm{PF}_{6}\right)$ shown in Figure $3 .{ }^{11}$ In solution, the degree of dissociation between the aryl moieties and the anion depends on both the polarity of the solvent and counter anion. ${ }^{12}$ However, it is believed that the dissociated species more-or-less retains its T-shape structure even in solution. ${ }^{13}$

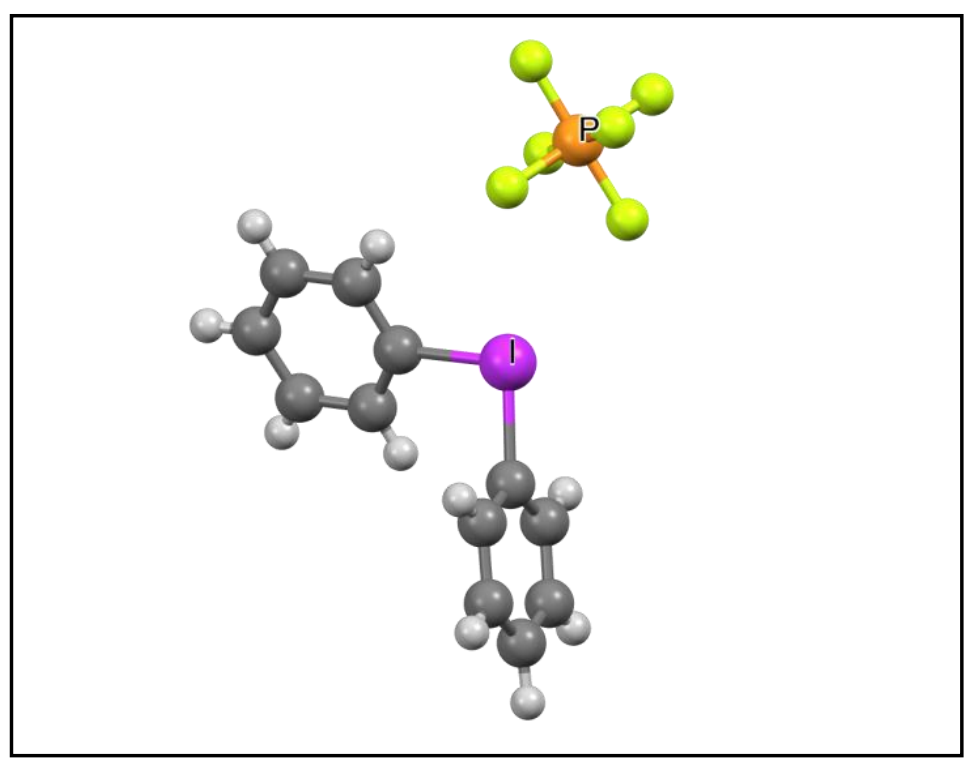

Figure 3. Crystal structure of diphenyliodonium hexafluorophosphate ${ }^{11}$

The electrophilic character of diaryliodonium salts makes them excellent candidates for arylation reactions. While symmetric salts are generally preferred to reduce selectivity issues in those conditions, numerous methods have now emerged demonstrating how unsymmetrical salts can expand the scope of such transformations. ${ }^{9}$ A non-transferable group, termed a "dummy 
group", is then designed for specific applications. Of those, 2,4,6-trimethoxyphenyl (TMP) has been proven to be efficient in metal-free reactions designed in the Stuart Group, and was exclusively chosen for this study. ${ }^{14}$

While studying chemical bonding in 1923, Gilbert N. Lewis defined an acidic substance as "one which can employ a lone pair from another molecule in completing the stable group of one

of its own atoms". "In other words", continues Lewis, "the basic substance furnishes a pair of electrons for a chemical bond, the acid substance accepts such a pair" ${ }^{15}$ A century later, species capable of accepting an electron pair are commonly termed Lewis acids in honor of his work. Lewis acids, in other words, are electron-deficient (electrophilic) species. The high electrondeficiency of diaryliodonium salts then naturally classifies them as Lewis acids. However, while several studies have focused on the electrophilic nature of these T-shaped complexes, ${ }^{13,16}$ very few, if any, have focused in a systematic way on the potential Lewis acidity that they may display.

This work aims to narrow the gap of knowledge surrounding the Lewis acidity of diaryliodonium salts. More specifically, the focus will be on quantifying the Lewis acidity of unsymmetrical aryl(2,4,6-trimethoxyphenyl) iodonium salts, or aryl(TMP)iodonium salts, to further the understanding of the reactive properties of these novel reagents. Additionally, a particular emphasis will be given on comparing phenyl(TMP)iodonium salts bearing different counter anions. It has been observed that counter anion identity greatly influences reactivity in arylation reactions, ${ }^{17}$ yet no information is available regarding how to select that critical component. Hence, this study also aims to help enhance rational selection of these counter anions.

\subsection{Objective}

To quantify the Lewis acidity of unsymmetrical aryl(2,4,6-trimethoxyphenyl) iodonium salts and explore the extent to which counter anion identity influences Lewis acidity. 


\subsection{Relevance}

Over $90 \%$ of current small molecules $(<550$ M.W.) of active ingredients in drugs contain aromatic rings ${ }^{18,19}$ and synthesis of those aryl compounds has therefore become a common necessity in the pharmaceutical industry. Similarly, a recent compilation of herbicides revealed that $90 \%$ of herbicides' active ingredients contain an aromatic ring as well. ${ }^{20}$ Synthesis of aryl compounds is primarily accomplished by aromatic substitution reactions such as classical nucleophilic aromatic substitution $\left(\mathrm{S}_{\mathrm{N}} \mathrm{Ar}\right)^{21}$ and contemporary transition metal catalysis. ${ }^{22}$ Due to the high cost and technical difficulty of metal-catalyzed arylations, research efforts have been focused on metal-free arylations which are severely limited by their reaction mechanism (i.e., $\left.\mathrm{S}_{\mathrm{N}} \mathrm{Ar}\right)$.

Previous data has supported the hypothesis that the iodonium moiety of diaryliodonium salts is one of few non-metals that can achieve a reductive elimination-like pathway to form arylnucleophile bonds and this may be exploited toward broader metal-free aromatic substitution. ${ }^{14}$ Diaryliodonium salts are novel reagents for metal-free arylation of nucleophiles, and the use of unsymmetrical aryl(2,4,6-trimethoxyphenyl)iodonium salts in reaction development is an underexplored area with significant potential.

The structure and reactivity of these hypervalent iodine compounds are generally similar to that of transition metal derivatives. ${ }^{4}$ In particular, hypervalent iodine reagents are often used in ligand exchange, oxidative addition, reductive elimination and ligand coupling reactions. However, while metals are often environmentally toxic, expensive and scarce, iodine benefits from being environmentally benign and relatively inexpensive. In the last decade, crude iodine has been sold around \$20-100 per kilogram, which is orders of magnitude cheaper than platinum, palladium, osmium and other commonly used metals. ${ }^{23}$ The estimated worldwide reserves of iodine are about 
15 million metric tons, located mainly in Chile and Japan, with an annual production of about 30,000 tons. ${ }^{23}$ Diaryliodonium salts hence represent environmentally sustainable alternatives to heavy metals.

The use of diaryliodonium salts in synthetic organic chemistry has risen sharply in the last decade due to the emergence of synthetic pathways and their discovery as effective metal-free arylation reagents. ${ }^{13,16}$ As air- and moisture-stable solid compounds, diaryliodonium salts are easy to use. Their low toxicity, good selectivity and high reactivity make them perfect candidates in a wide variety of transformations under both metal-free and metal-catalyzed conditions. The tremendous progress made over the past 20 years regarding aryl transfer with diaryliodonium salts implies that their electron-deficient character has been widely accepted. Amongst other things, this indicates that they are strong electron-acceptor candidates. Yet, their potential as Lewis acids has not been extensively studied so far. A thorough search of the relevant literature yielded only one study that made use of diaryliodonium salts as Lewis acid catalyst to promote a reaction, ${ }^{24}$ which emphasizes the lack of knowledge in this area. Although a few reports proposed that diaryliodonium salts act as Lewis acids prior to oxidation processes, ${ }^{25,26}$ hypervalent iodine reagents are greatly underexploited in this context. There is a current gap of knowledge on how diaryliodonium salts compare to common Lewis acids.

Recent work by Labattut et al. ${ }^{27}$ aimed to quantify the Lewis acidity of iodine(III) species, including some examples of diphenyliodonium salts $\left(\mathrm{Ph}_{2} \mathrm{I}-\mathrm{PF}_{6}\right.$ and $\left.\mathrm{Ph}_{2} \mathrm{I}-\mathrm{OTs}\right)$. However, unsymmetrical aryl(TMP)iodonium salts are more attractive than their symmetrical counterparts because they produce a less wasteful, reusable auxiliary iodine in arylation reactions. They have also been quite underexploited in literature, as $90 \%$ of metal-free methods using diaryliodonium salts listed on PubMed.gov (last 5 years) made use of the more wasteful symmetrical salts. 
Although unsymmetrical diaryliodonium salts are now easier to prepare, they are seldom used in a routine way. Gathering additional knowledge will encourage the organic synthesis community to use this scarcely explored but highly efficient and greener alternative.

Building on previous and ongoing research in the Stuart Group, this study will focus primarily on aryl(TMP)iodonium salts. The effect of different counter anions will be an emphasis in the characterization of Lewis acidity. The counter anion component of these salts is reversibly displaced by nucleophiles and appears to often exert dramatic effects on reactivity. As such, it is a critical component to explore, and determining its effect on Lewis acidity may lead to insights on reactivity. In particular, the triflate (OTf), trifluoroacetate (TFA) and tetrafluoroborate $\left(\mathrm{BF}_{4}\right)$ counter anions were selected to be studied due to their important presence in literature. Additionally, the hexafluorophosphate $\left(\mathrm{PF}_{6}\right)$ counter anion was studied for a closer comparison with the values found by Labattut et al. ${ }^{27}$, while the tosylate (OTs) anion was chosen per its popularity within the Stuart Group as an efficient and often high-yielding compound. 


\section{Methods}

\subsection{Synthesis}

The iodonium salts used in this study were made according to the one-pot synthesis procedure described by Seidl et. al. ${ }^{14}$ First, aryl(2,4,6-trimethoxyphenyl)iodonium tosylate salts were produced from the two-steps reaction shown in Figure 4 below.

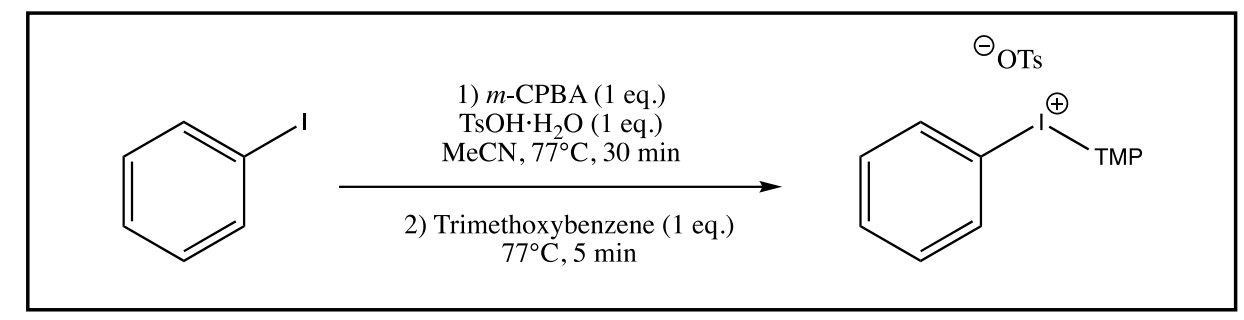

Figure 4. Synthesis of aryl(2,4,6-trimethoxyphenyl)iodonium tosylate

The procedure combines iodobenzene $(1$ eq. $)$ and acetonitrile $(5 \mathrm{~mL})$ in a $50 \mathrm{~mL}$ roundbottom flask along with a magnetic stir bar. Toluenesulfonic acid (1 eq.) is then added in one portion, followed by $m$-CPBA ( 1 eq.). The reaction mixture is set into a $77^{\circ} \mathrm{C}$ oil bath, attached to a reflux condenser and stirred vigorously for 30 minutes. One equivalent of 1,3,5trimethoxybenzene is then added in one portion and stirred for an additional 5 minutes. After removing the reaction from heat, the crude residue is triturated with diethyl ether. The precipitate is then isolated by vacuum filtration, washed by slurry filtration with diethyl ether $(3 \times 20 \mathrm{~mL})$ and dried under high vacuum before being collected.

The phenyl(TMP)tosylate salt needed for the next synthesis step was prepared according to the above procedure using $1.7 \mathrm{~mL}$ of iodobenzene, $2.6010 \mathrm{~g}$ of $m$-CPBA and $2.8590 \mathrm{~g}$ of $\mathrm{TsOH} \cdot \mathrm{H}_{2} \mathrm{O}$ in $15 \mathrm{~mL}$ of acetonitrile. Next, $2.5720 \mathrm{~g}$ of trimethoxybenzene were added to achieve a $74 \%$ yield $(6.0661 \mathrm{~g})$. Identity and purity of this compound was confirmed through ${ }^{1} \mathrm{H}$ NMR spectroscopy (400 MHz, DMSO- $d_{6}$ ), yielding chemical shifts consistent with literature. ${ }^{14}$ 
Similarly, the para-tBu-phenyl(TMP) tosylate salt studied was synthesized using $3.6 \mathrm{~mL}$ of 4-tert-butyliodobenzene, $3.4986 \mathrm{~g}$ of $m$-CPBA and $4.0777 \mathrm{~g}$ of $\mathrm{TsOH} \cdot \mathrm{H}_{2} \mathrm{O}$ in $20.05 \mathrm{~mL}$ of acetonitrile. Then, $3.6709 \mathrm{~g}$ of trimethoxybenzene were added to achieve a $68 \%$ yield $(8.2859 \mathrm{~g})$. Once again, the ${ }^{1} \mathrm{H}$ NMR spectra (400 MHz, DMSO- $d_{6}$ ) resembled literature values. ${ }^{14}$

Next, counter anion exchange was performed to synthesize all other salts used in this study as depicted in Figure 5 below.

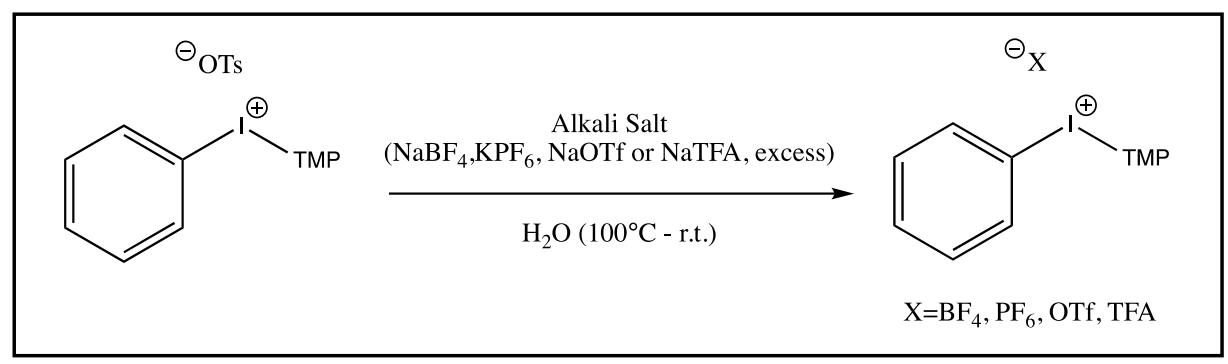

Figure 5. Counter anion exchange for synthesis of aryl(TMP) iodonium salts

Here, one equivalent of the previously synthesized tosylate iodonium salt was added to 50 $\mathrm{mL}$ of boiling water. If the salt did not dissolve after a few minutes, methanol was added portionwise. Excess alkali salt containing the target anion was then added in excess (100 equivalents for

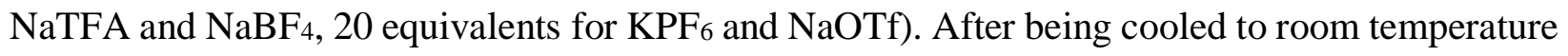
and chilled in an ice-bath, the mixture was suction filtered and washed by slurry filtration with water $(3 \times 30 \mathrm{~mL})$ and then dried under suction for 10-20 minutes. Finally, the sample was washed by slurry filtration with diethyl ether $(3 \times 30 \mathrm{~mL})$ and left to dry under high vacuum before being collected.

The $\mathrm{BF}_{4}$ salt was prepared using $1.3593 \mathrm{~g}$ of tosylate salt and $27.5010 \mathrm{~g}$ of $\mathrm{NaBF}_{4}$ in 50 $\mathrm{mL} \mathrm{H}_{2} \mathrm{O}$ to achieve $58 \%$ yield $(0.668 \mathrm{~g})$. The OTf salt made use of $1.3575 \mathrm{~g}$ of tosylate salt and $4.3046 \mathrm{~g}$ of $\mathrm{NaOTf}$ in $50 \mathrm{~mL} \mathrm{H} \mathrm{H}_{2} \mathrm{O}$ to achieve $71 \%$ yield $(0.9237 \mathrm{~g})$. The TFA salt was done using $1.1622 \mathrm{~g}$ of tosylate salt and $29.2826 \mathrm{~g}$ of NaTFA in $50 \mathrm{~mL} \mathrm{H}_{2} \mathrm{O}$ to achieve $62 \%$ yield $(0.6215 \mathrm{~g})$. Lastly, the $\mathrm{PF}_{6}$ salt was synthesized from $1.2541 \mathrm{~g}$ of tosylate salt and $8.663 \mathrm{~g}$ of $\mathrm{KPF}_{6}$ in $50 \mathrm{~mL}$ 
and reached $80 \%$ yield (1.0086 g). All of these compounds were studied using ${ }^{1} \mathrm{H}$ NMR spectroscopy and generated the expected chemical shifts reported in literature. ${ }^{14}$

As discussed previously, salts were chosen based on the reduced waste potential of unsymmetrical salts and the practical and useful applications of the 2,4,6-trimethoxyphenyl dummy group. All salts studied are depicted in Figure 6 below.

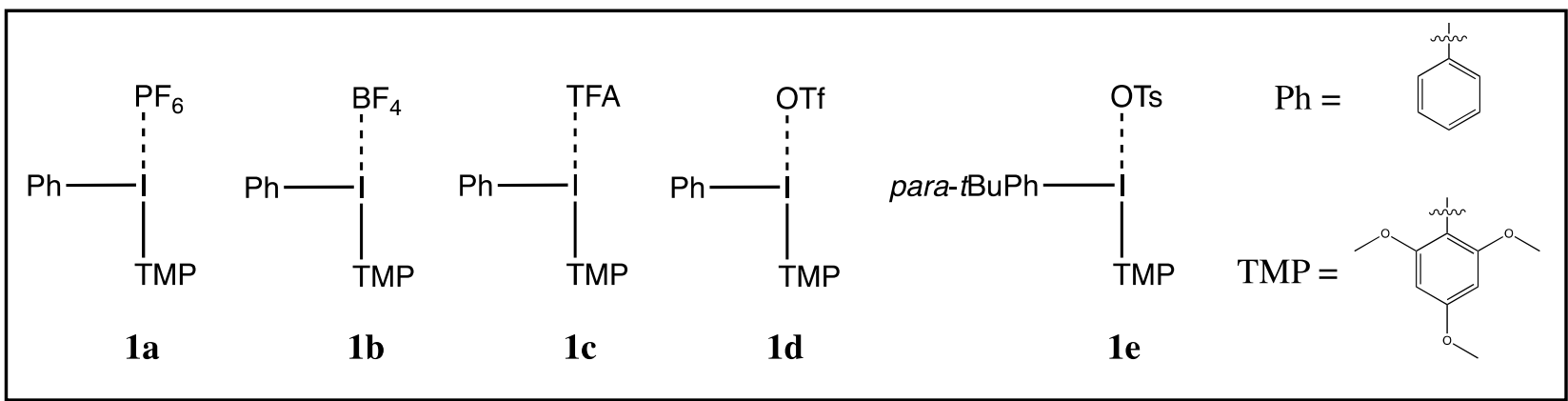

Figure 6. Aryl(TMP)iodonium salts studied 


\subsection{Gutmann-Beckett Method}

Nowadays, quantifying acidity as defined by the Brønsted-Lowry model is conveniently done through a widely accepted universal $\mathrm{pK}_{\mathrm{a}}$ scale. Although no universal scale exists to measure Lewis acidity, numerous methods have emerged in which quantifying Lewis acidity is made possible.

One of the most common and practical strategies used has been the Gutmann-Becket method, ${ }^{28,29}$ in which the NMR chemical shift displacements $(\Delta \delta)$ from the complexation of a Lewis base probe with the studied Lewis acid is measured. The Gutmann-Beckett method has been successfully used for a large and varied range of Lewis acids and is thus widely recognized in the field. Its main advantage is that it results in a single displacement that is easily measured by ${ }^{31} \mathrm{P}\left\{{ }^{1} \mathrm{H}\right\}$ NMR.

This specific procedure uses triethylphosphine oxide (TPO) as a Lewis base probe, measured using ${ }^{31} \mathrm{P}$ decoupled ${ }^{1} \mathrm{H}$ NMR. The structure of TPO is such that steric effects are minimal, thereby facilitating comparison of the studied salts with other Lewis acids. A $400 \mathrm{MHz}$ spectrometer was

used to measure the ${ }^{31} \mathrm{P}$ chemical shift displacement between the TPO-Lewis acid adduct and free TPO. Finally, since the classic reference compound in ${ }^{31} \mathrm{P}$ NMR experiment is usually $85 \% \mathrm{H}_{3} \mathrm{PO}_{4}$ in $\mathrm{H}_{2} \mathrm{O}(0 \mathrm{ppm})$, a capillary insert of phosphoric acid $\left(\mathrm{H}_{3} \mathrm{PO}_{4}\right)$ was placed in all samples (Figure 7). Having this external standard ensured precise calibration of each spectrum. 


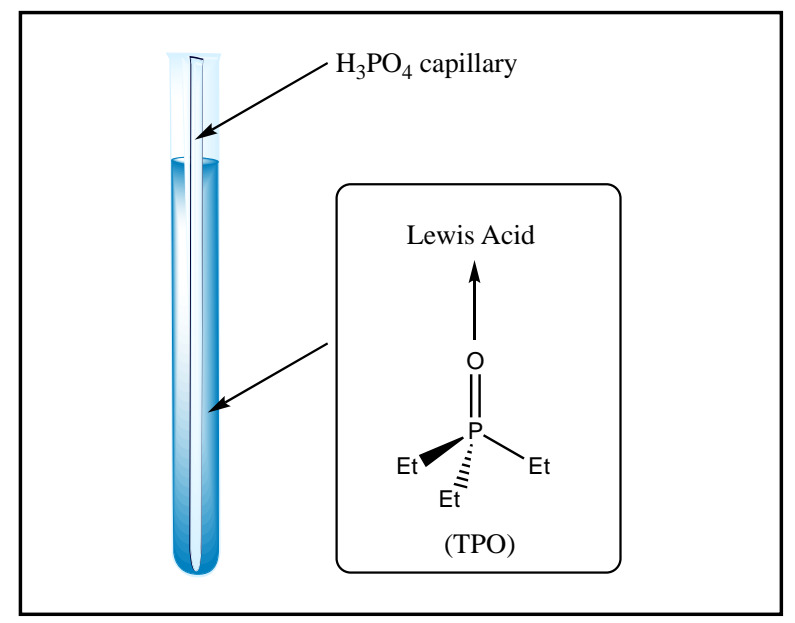

\section{Figure 7. Experimental setup of NMR sample}

As the actual binding affinity of TPO to the studied unsymmetrical aryl $(2,4,6$ trimethoxyphenyl)iodinium salts was unknown, titrations were performed. Nonlinear regression was then used to estimate association constants as described next. Deuterated acetonitrile $\left(\mathrm{CD}_{3} \mathrm{CN}\right)$ was found to be a suitable solvent by Labattut et al. and was hence used for titrations. Most titrations were performed with iodonium salt equivalency values of $0,0.2,0.4,0.6,0.8,1,1.5,2$, 4, 6, 8, 16 and 30. A few exceptions appeared, as described in the Results section, due to the limits caused by insolubility issues at high concentrations and a certain minimum concentration needed to bypass the NMR sensitivity threshold.

It is also important to note that the highly hygroscopic nature of TPO combined with the volatility of the solvents used required all samples to be tightly sealed and examined promptly. Although TPO may be delicate to worth with, it fulfils the important requirements needed for reliable measurements as described by Gutmann. ${ }^{28}$ That is, the ${ }^{31} \mathrm{P}$ nucleus is remote from the actual place of interaction, namely the oxygen atom, thus eliminating unwanted contact contributions to the chemical shift measured. This interaction site is always well-defined, and the incorporation of ethyl groups guarantees efficient electronic shielding without steric hindrance. TPO is also a very strong base, which put together with the partial double bond character of the 
P-O bond, assures a high sensitivity of measurement. Finally, TPO is soluble in all kinds of solvents and does not decompose easily.

\subsection{Data Analysis}

In order to carefully analyze the data collected from the Gutmann-Beckett method, it is necessary to explore its corresponding binding model in detail. As described previously, this titration approach uses a Lewis acid (guest) that is gradually added to the Lewis base (host) while the specific chemical resonance is monitored via NMR spectroscopy.

In this model, the one-to-one binding of two elements was explored. This simple 1:1 equilibria system can be represented by a host $(\mathrm{H})$ - guest $(\mathrm{G})$ relationship as $H+\mathrm{G} \rightleftharpoons \mathrm{HG}$. Quantifying the interaction between those components is then a matter of determining the association constant, $K_{a}$, defined in this system by the ratio of the products' and reactants' concentrations as shown in equation (1):

$$
K_{a}=\frac{[\mathrm{HG}]}{[\mathrm{H}][\mathrm{G}]}
$$

While $[\mathrm{H}]$ and $[\mathrm{G}]$ are known for each sample, the concentration of the complex $[\mathrm{HG}]$ is usually not possible to measure. Hence, other methods must be used to indirectly determine the concentration of the complex. By realizing the fact that NMR shifts are correlated to the concentration of the complex, a mathematical model can be built using only the known concentrations and the observed chemical shift to estimate the association constant $K_{a}$.

Although many linear models have been created in the past, such as the popular BenesiHildebrand plot $^{30}$ (known as Lineweaver-Burk plot in enzyme kinetics ${ }^{31}$ ), more accurate results can be obtained by using modern computer technology to evaluate nonlinear regression models. The derivation of those models results from a few observations about the properties of our experiment, along with a little bit of algebra, as detailed next. 
Since matter can neither be created nor destroyed, the law of conservation applies to each component of the equilibrium. The host species is present in solution as an individual species as well as inside a host-guest complex. Hence the total concentration of host, $[\mathrm{H}]_{0}$, must be equal to the sum of those two states. The same applies to the guest species, which can then be mathematically translated into the following mass balance equations:

$$
\begin{aligned}
{[\mathrm{H}]_{0} } & =[\mathrm{H}]+[\mathrm{HG}] \\
{[\mathrm{G}]_{0} } & =[\mathrm{G}]+[\mathrm{HG}]
\end{aligned}
$$

Rewriting (2) and (3) in terms of $[\mathrm{H}]$ and $[\mathrm{G}]$ respectively yields $[\mathrm{H}]=[\mathrm{H}]_{0}-[\mathrm{HG}]$ and $[G]=[G]_{0}-[H G]$. Hence, the definition of $K_{a}$ from (1) can be rewritten based on $[H]_{0}$ and $[\mathrm{G}]_{0}$ to yield a quadratic equation as shown here:

$$
\begin{array}{rlrl}
K_{a} & =\frac{[\mathrm{HG}]}{[\mathrm{H}][\mathrm{G}]}=\frac{[\mathrm{HG}]}{\left([\mathrm{H}]_{0}-[\mathrm{HG}]\right)\left([\mathrm{G}]_{0}-[\mathrm{HG}]\right)} & & \text { by substitution, } \\
& =\frac{[\mathrm{HG}]}{[\mathrm{H}]_{0}[\mathrm{G}]_{0}-[\mathrm{H}]_{0}[\mathrm{HG}]-[\mathrm{HG}][\mathrm{G}]_{0}+[\mathrm{HG}]^{2}} & & \text { expanding. } \\
\text { So, } \frac{1}{K_{a}} & =\frac{[\mathrm{HG}]^{2}-[\mathrm{H}]_{0}[\mathrm{HG}]-[\mathrm{HG}][\mathrm{G}]_{0}+[\mathrm{H}]_{0}[\mathrm{G}]_{0}}{[\mathrm{HG}]} & \text { inverting, } \\
& =[\mathrm{HG}]-[\mathrm{H}]_{0}-[\mathrm{G}]_{0}+\frac{[\mathrm{H}]_{0}[\mathrm{G}]_{0}}{[\mathrm{HG}]} & \text { simplifying, } \\
\text { or } & {[\mathrm{HG}]-[\mathrm{H}]_{0}-[\mathrm{G}]_{0}+\frac{[\mathrm{H}]_{0}[\mathrm{G}]_{0}}{[\mathrm{HG}]}+\frac{1}{K_{a}}=0} & \text { by adding } \frac{1}{K_{a}} .
\end{array}
$$

After multiplying the above equation by [HG] , a quadratic equation of the form $a X^{2}+$ $b X+c=0$ is obtained, where $X=[\mathrm{HG}], a=1, b=-\left([\mathrm{H}]_{0}+[\mathrm{G}]_{0}+\frac{1}{\mathrm{~K}_{\mathrm{a}}}\right)$, and $c=[\mathrm{H}]_{0}[\mathrm{G}]_{0}$ as shown in equation (4):

$$
[\mathrm{HG}]^{2}-[\mathrm{HG}]\left([\mathrm{H}]_{0}+[\mathrm{G}]_{0}+\frac{1}{K_{a}}\right)+[\mathrm{H}]_{0}[\mathrm{G}]_{0}=0
$$


The solution of equation (4) is then given by the positive root from the quadratic formula $X=\frac{1}{2 a}\left(-b \pm \sqrt{b^{2}-4 a c}\right)$, or in this case:

$$
[\mathrm{HG}]=\frac{1}{2}\left[\left([\mathrm{G}]_{0}+[\mathrm{H}]_{0}+\frac{1}{K_{a}}\right)-\sqrt{\left(\left([\mathrm{G}]_{0}+[\mathrm{H}]_{0}+\frac{1}{K_{a}}\right)^{2}-4[\mathrm{H}]_{0}[\mathrm{G}]_{0}\right.}\right]
$$

This relationship is particularly useful and powerful, as [HG] can now be estimated based on only one unknown, $K_{a}$.

Similarly to the mass balance equations described in (2) and (3), the chemical shift observed via NMR spectroscopy $\left(\delta_{\text {obs }}\right)$ is composed of the sum of individual species present as a function of mole fractions, ${ }^{32}$ yielding the following relationship:

$$
\delta_{\mathrm{obs}}=\delta_{\mathrm{H}} f_{\mathrm{H}}+\delta_{\mathrm{G}} f_{\mathrm{G}}+\delta_{\mathrm{HG}} f_{\mathrm{HG}}
$$

where a mole fraction $f_{\mathrm{X}}$ is defined as the ratio of the species' concentration, $[\mathrm{X}]$, over its total concentration $[\mathrm{X}]_{0}$.

Further simplifications can be made by using the fact that our free guest (Lewis acid, or iodonium salt) is "silent". That is, the guest species does not contain a ${ }^{31} \mathrm{P}$ atom, and hence will not play a part in the ${ }^{31} \mathrm{P}$ NMR signal measured. In addition, the total mole fraction of host $\left(f_{\mathrm{H}_{0}}\right)$ is composed of the mole fraction of unbound host $\left(f_{\mathrm{H}}\right)$ and of bound host $\left(f_{\mathrm{HG}}\right)$. By definition of mole fraction, the total $f_{\mathrm{H}_{0}}$ is also equal to 1 . It hence holds that $f_{\mathrm{H}_{0}}=f_{\mathrm{H}}+f_{\mathrm{HG}}=1$, or $f_{\mathrm{H}}=1-$ $f_{\mathrm{HG}}$. Thus, equation (6) can be simplified by using those expressions and then substituting $f_{\mathrm{HG}}=$ $\frac{[\mathrm{HG}]}{[\mathrm{H}]_{0}}$ as follows: 


$$
\begin{aligned}
& \delta_{\mathrm{obs}}=\delta_{\mathrm{H}} f_{\mathrm{H}}+\delta_{\mathrm{HG}} f_{\mathrm{HG}} \quad \text { assuming guest is silent, } \\
& =\delta_{\mathrm{H}}\left(1-f_{\mathrm{HG}}\right)+\delta_{\mathrm{HG}} f_{\mathrm{HG}} \quad \text { by substitution, } \\
& =\delta_{\mathrm{H}}-\delta_{\mathrm{H}} f_{\mathrm{HG}}+\delta_{\mathrm{HG}} f_{\mathrm{HG}} \quad \text { expanding, } \\
& =\delta_{\mathrm{H}}+f_{\mathrm{HG}}\left(\delta_{\mathrm{HG}}-\delta_{\mathrm{H}}\right) \quad \text { factoring } f_{\mathrm{HG}} \text { out, } \\
& \delta_{\mathrm{obs}}=\delta_{\mathrm{H}}+\frac{[\mathrm{HG}]}{[\mathrm{H}]_{0}}\left(\delta_{\mathrm{HG}}-\delta_{\mathrm{H}}\right) \quad \text { by substitution. }
\end{aligned}
$$

By introducing new notation, this equation can be simplified one last time to yield the linear relationship shown in equation (7):

$$
\Delta \delta_{\text {calc }}=\Delta \delta_{\max }\left(\frac{[\mathrm{HG}]}{[\mathrm{H}]_{0}}\right)
$$

where $\Delta \delta_{\text {calc }}=\delta_{\text {obs }}-\delta_{\mathrm{H}}$ represents the theoretical, calculated chemical shift difference, and $\Delta \delta_{\max }=\delta_{\mathrm{HG}}-\delta_{\mathrm{H}}$ represents the maximal chemical shift observed.

In practice, Microsoft ${ }^{\circledR}$ Excel 2018's solver was used to vary $K_{a}$ and $\Delta \delta_{\max }$ values simultaneously in order to minimize the residual sum of squares (RSS) between calculated and observed values, $\sum\left(\Delta \delta_{\text {calc }}-\Delta \delta_{\text {obs }}\right)^{2}$. Both $K_{a}$ and $\Delta \delta_{\text {max }}$ were given an initial value to determine the theoretical chemical shift difference $\Delta \delta_{\text {calc }}$ which was computed using equation (7). In that expression, equation (5) was used to estimate [HG]. Additionally, the observed chemical shift difference $\Delta \delta_{\text {obs }}$ directly came from the distance between chemical shifts observed on NMR spectra.

\subsection{Obstacles}

Lastly, it is important to explore the obstacles and limitations of this study. Of those, solvent choice has been a clear challenge. The first solvent limitation naturally occurs due to the nature of NMR spectroscopy. As explained previously, ${ }^{31} \mathrm{P}$ decoupled ${ }^{1} \mathrm{H}$ NMR was used to yield the most 
accurate results. Decoupling was used to minimize spectrum noise from the interactions between hydrogen and phosphorus. Usually, NMR experiments coupled to ${ }^{1} \mathrm{H}$ require that the solvent used must have little to no ${ }^{1} \mathrm{H}$ atoms to ensure that it does not interfere with the NMR spectrum of the sample. This requirement can be bypassed through the use of deuterated solvents containing deuterium $\left({ }^{2} \mathrm{H}\right.$, or D) instead of hydrogen ${ }^{1} \mathrm{H}$, or solvents that do not contain hydrogen in the first place, such as carbon tetrachloride $\left(\mathrm{CCl}_{4}\right)$. However, these solvents are often expensive, toxic, or not commercially available. Since ${ }^{1} \mathrm{H}$ interactions were removed from the spectrum via decoupling, deuterated solvents were not necessary in this study. However, NMR spectroscopy generally functions better under these conditions as the machine most easily identifies and locks the signal of the given sample when deuterated solvents are used. Solvent choice was thus limited to the most inexpensive and readily available deuterated solvents.

Diaryliodonium salts, as polar species, dissolve well in polar solvents such as methanol. However, such coordinating solvents cannot be used as they may interfere with the Lewis acidbase binding. More specifically, solvent molecules may compete with TPO and thus render measurements meaningless. Hence, non-polar, non-coordinating solvents must be used. A clear issue then arises, as most diaryliodonium salts do not readily dissolve in these types of solvents.

Additional obstacles appear as the properties of diaryliodonium salts from the same family differ greatly from one counter anion to the next. For example, while phenyl-(2,4,6trimethoxyphenyl)-iodonium triflate dissolves readily in acetonitrile, phenyl-(2,4,6trimethoxyphenyl)-iodonium tosylate does not. Similarly, reactivity trends across different counter anions have been observed but are not yet well understood. ${ }^{17}$ By exploring the same family of diaryliodonium salts across different counter anions, this work aims to shine some light on the role that this entity plays in reaction mechanisms. Due to the different solubility properties of these 
compounds, different families of salts had to be used. For the $\mathrm{PF}_{6}, \mathrm{BF}_{4}$, TFA and OTf counter anions, phenyl(TMP)iodonium was used. However, para-tBuphenyl(TMP)iodonium was selected in order to study the tosylate (OTs) counter anion due to the insolubility issues stated previously. 


\section{Results}

\subsection{Phenyl(TMP)Iodonium Hexafluorophosphate}

The diaryliodonium salt bearing the $\mathrm{PF}_{6}$ counter anion (1a) was studied in deuterated acetonitrile $\left(\mathrm{CD}_{3} \mathrm{CN}\right)$ with a TPO concentration of $20.64 \mathrm{mM}$. Figure 8 below illustrates the titration curve obtained experimentally. Note that $\Delta \delta_{\max }$ represents the asymptotical maximum chemical shift difference where the curve plateaus near $5 \mathrm{ppm}$. For raw data detailing the equivalents used and chemical shifts obtained, please refer to the Appendices.

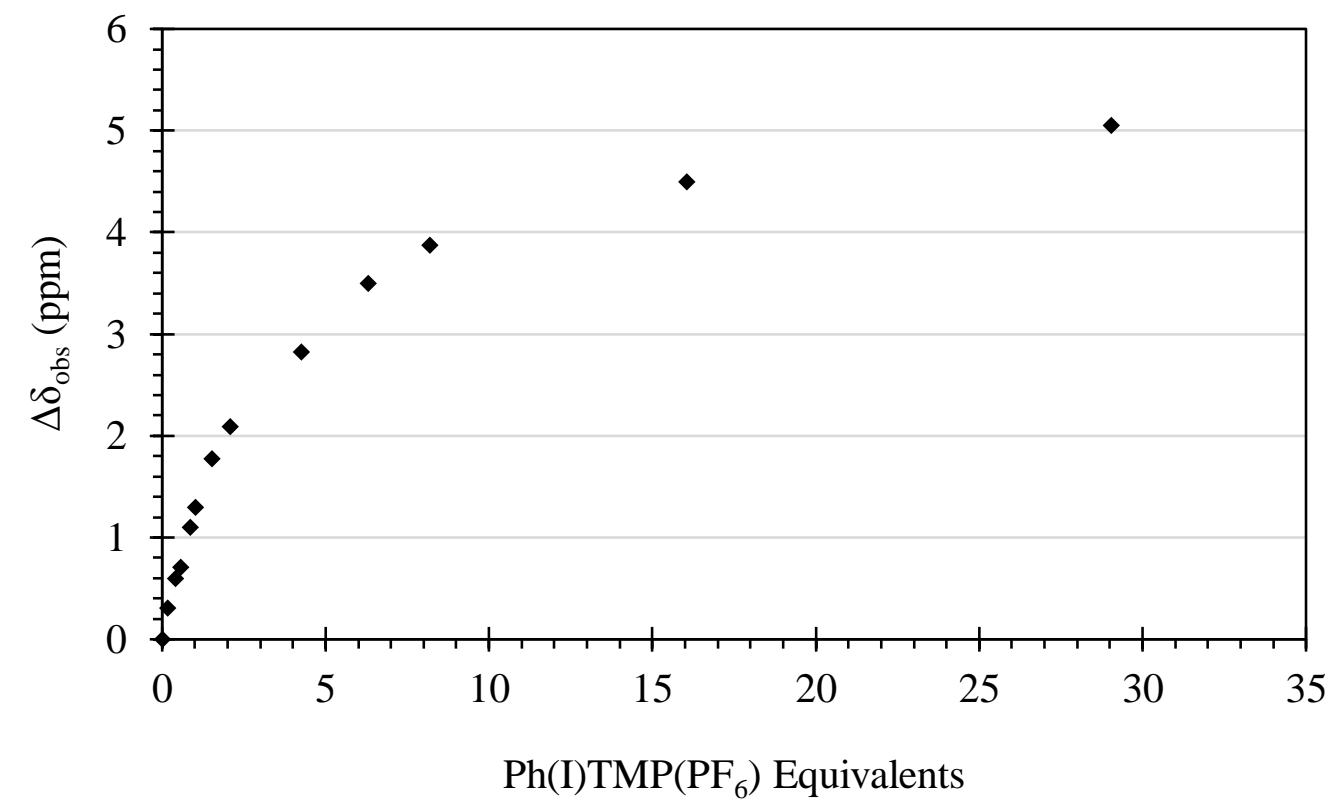

Figure 8. Experimental titration curve for $\mathrm{Ph}(\mathrm{I}) \mathrm{TMP}\left(\mathrm{PF}_{6}\right)$ in $\mathrm{CD}_{3} \mathrm{CN}$

After running the regression model, a more precise $\Delta \delta_{\max }$ value of $5.4012 \mathrm{ppm}$ was obtained, while the association constant was determined to be $K_{a}=17.06 \mathrm{M}^{-1}$.

This relationship can also be plotted in a more eye-friendly linear plot. Recall from equation (7) that $\Delta \delta_{\text {calc }}=\Delta \delta_{\max }\left(\frac{[\mathrm{HG}]}{[\mathrm{H}]_{0}}\right)$, or $\Delta \delta_{\text {calc }}=\Delta \delta_{\max }\left(f_{\mathrm{HG}}\right)$. Hence, $\Delta \delta_{\text {calc }}$ and $f_{\mathrm{HG}}$ have a 
linear relationship where $\Delta \delta_{\max }$ approximately represents the slope of the plotted line, as shown in Figure 9.

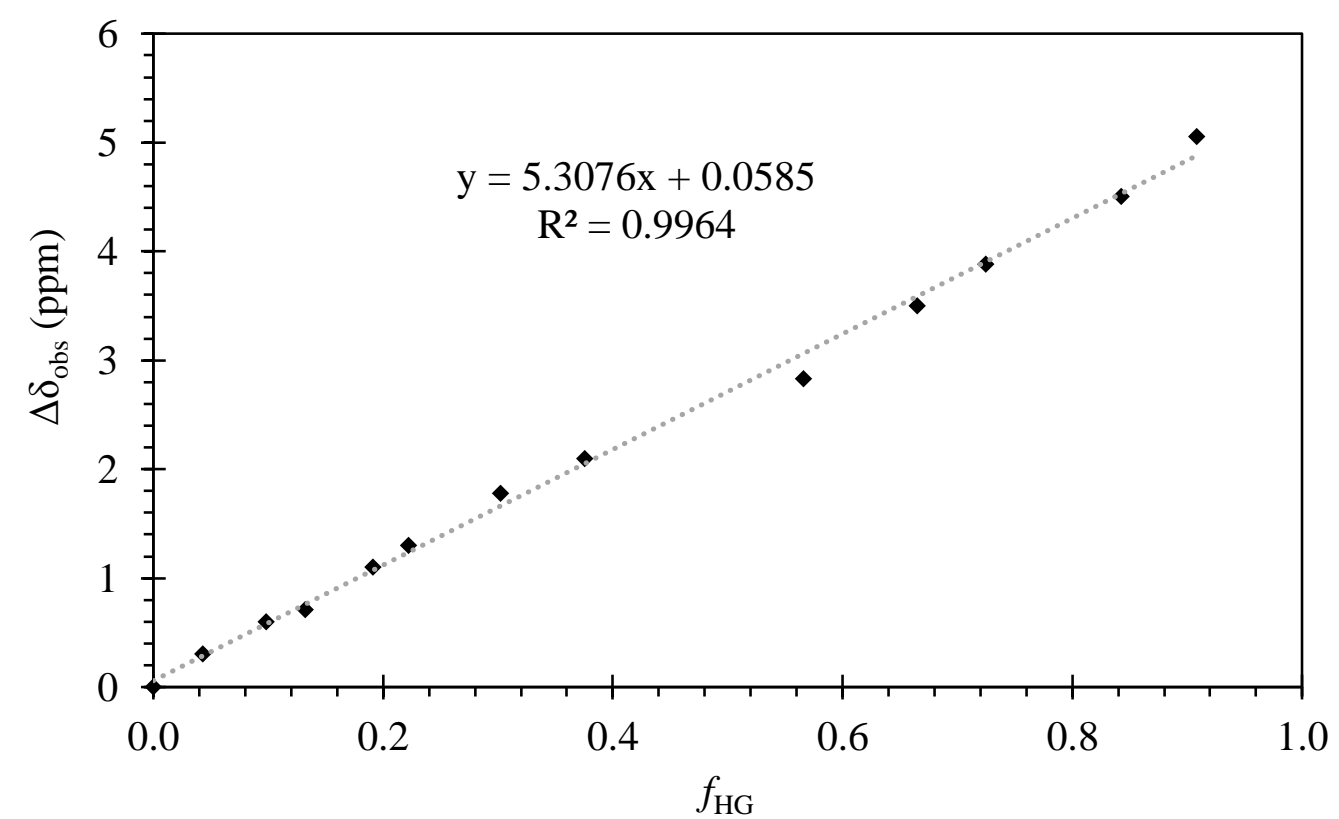

Figure 9. Linear plot for $\mathrm{Ph}(\mathrm{I}) \mathrm{TMP}\left(\mathrm{PF}_{6}\right)$ in $\mathrm{CD}_{3} \mathrm{CN}$

Note that the coefficient of determination, or $\mathrm{R}^{2}$ value, displayed in Figure 9 shows that $99.64 \%$ of the variability in the observed chemical shift difference, $\Delta \delta_{\text {obs }}$, is explained by its linear relationship with the mole fraction of host-guest complex, $f_{\mathrm{HG}}$. This high percentage value confirms, with a high degree of confidence, the validity of our model.

\subsection{Phenyl(TMP)Iodonium Tetrafluoroborate}

Similarly, the titration curve for the $\mathrm{BF}_{4}$ bearing salt $(\mathbf{1 b})$ is shown below in Figure 10 . This salt was also studied in deuterated acetonitrile, with a TPO concentration of $20.64 \mathrm{mM}$. The association constant was determined to be $K_{a}=32.99 \mathrm{M}^{-1}$ with a $\Delta \delta_{\max }$ value of $5.9092 \mathrm{ppm}$. 


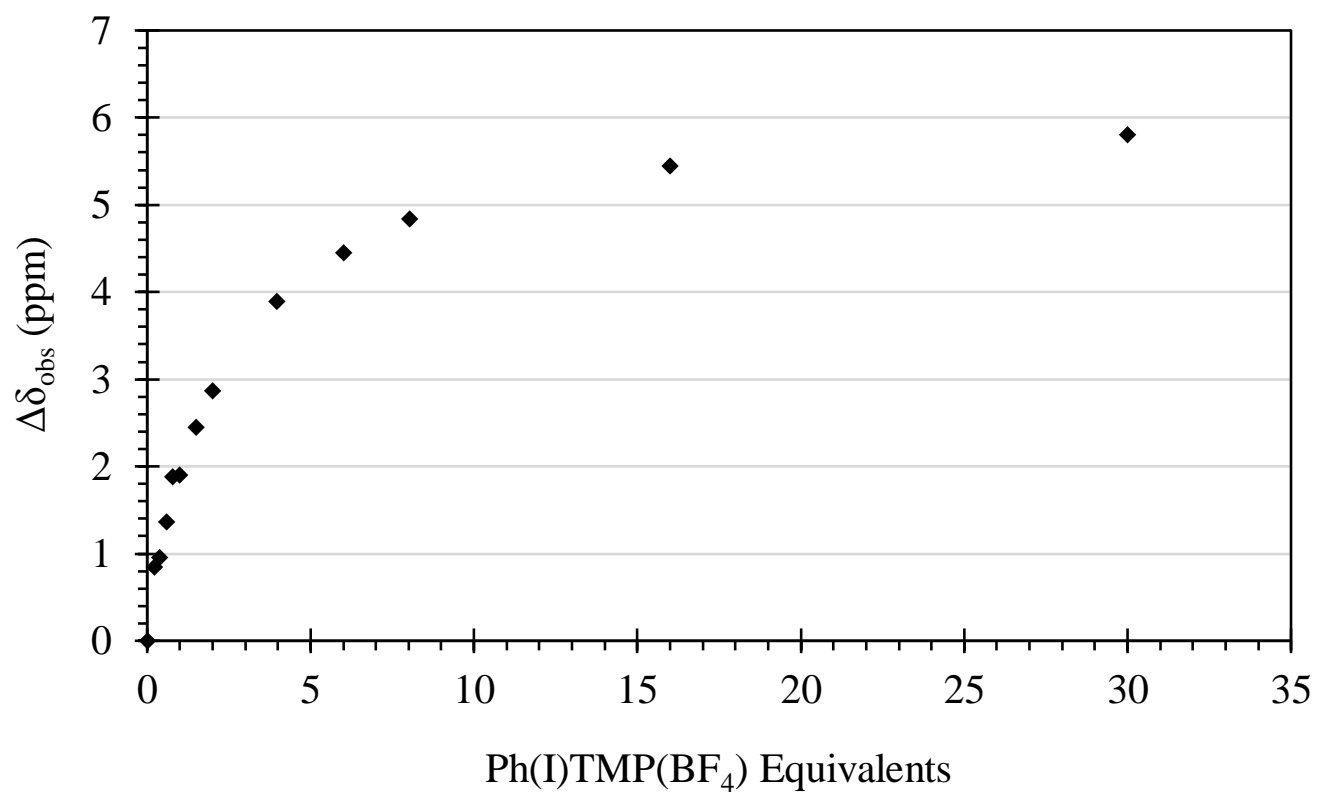

Figure 10. Experimental titration curve for $\operatorname{Ph}(\mathrm{I}) \mathrm{TMP}\left(\mathrm{BF}_{4}\right)$ in $\mathrm{CD}_{3} \mathrm{CN}$

Once again, the linear plot helps confirm our fit with a $\mathrm{R}^{2}$ value of 0.9933 as shown in Figure 11.

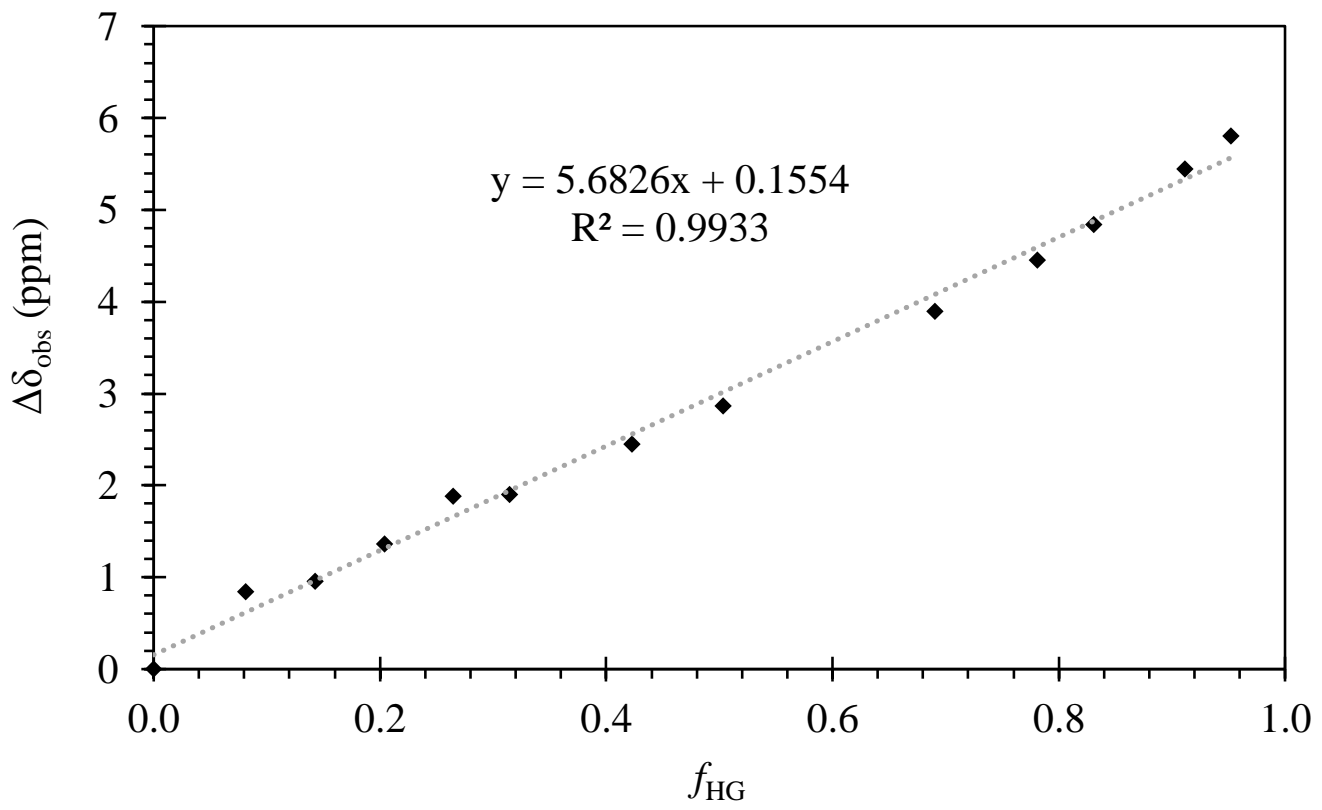

Figure 11. Linear plot for $\operatorname{Ph}(\mathrm{I}) \mathrm{TMP}\left(\mathrm{BF}_{4}\right)$ in $\mathrm{CD}_{3} \mathrm{CN}$ 


\subsection{Phenyl(TMP)Iodonium Trifluoroacetate}

The TFA salt (1c) is shown in Figure 12 and Figure 13. This salt was also studied in deuterated acetonitrile, with a TPO concentration of $20.16 \mathrm{mM}$. The association constant was determined to be $K_{a}=46.43 \mathrm{M}^{-1}$ and $\Delta \delta_{\max }=2.7056 \mathrm{ppm}$. Due to insolubility issues that arose above 15 equivalents, the last couple experiments resulted in lower $\Delta \delta_{\text {obs }}$ measurements than expected. Extrapolated values should hence be treated with caution, as indicated by the lower $\mathrm{R}^{2}$ value shown in Figure 13. Ignoring these data points results in a marginal increase in $K_{a}$ and $\Delta \delta_{\text {max }}$, but further data collection is needed to enhance the validity of these values. In particular, using a lower initial TPO concentration should yield the expected titration curve needed to extrapolate precise data.

Additionally, it must be noted that the $K_{a}$ value obtained from the mathematical model is clearly inconsistent with the data collected. As shown in Figure 12, the $\Delta \delta_{\max }$ recorded here was much smaller than values obtained for previous salts (2.5 ppm versus 5 to $6 \mathrm{ppm}$ ). Since $\Delta \delta_{\max } \propto$ $K_{a}$ (see equation (7)), a lower $\Delta \delta_{\max }$ should yield a lower $K_{a}$. In other words, the effect of the Lewis acid on the base was measured to be much weaker, indicating a lower Lewis acidity of the compound studied. Hence, the association constant obtained is disproportionately large and does not reflect the relative acidity of phenyl(TMP)iodonium trifluoroacetate. Based on the $\Delta \delta_{\text {max }}$ value obtained, this TFA salt can be classified as having a lower Lewis acidity than the $\mathrm{PF}_{6}$ and $\mathrm{BF}_{4}$ salts above. 


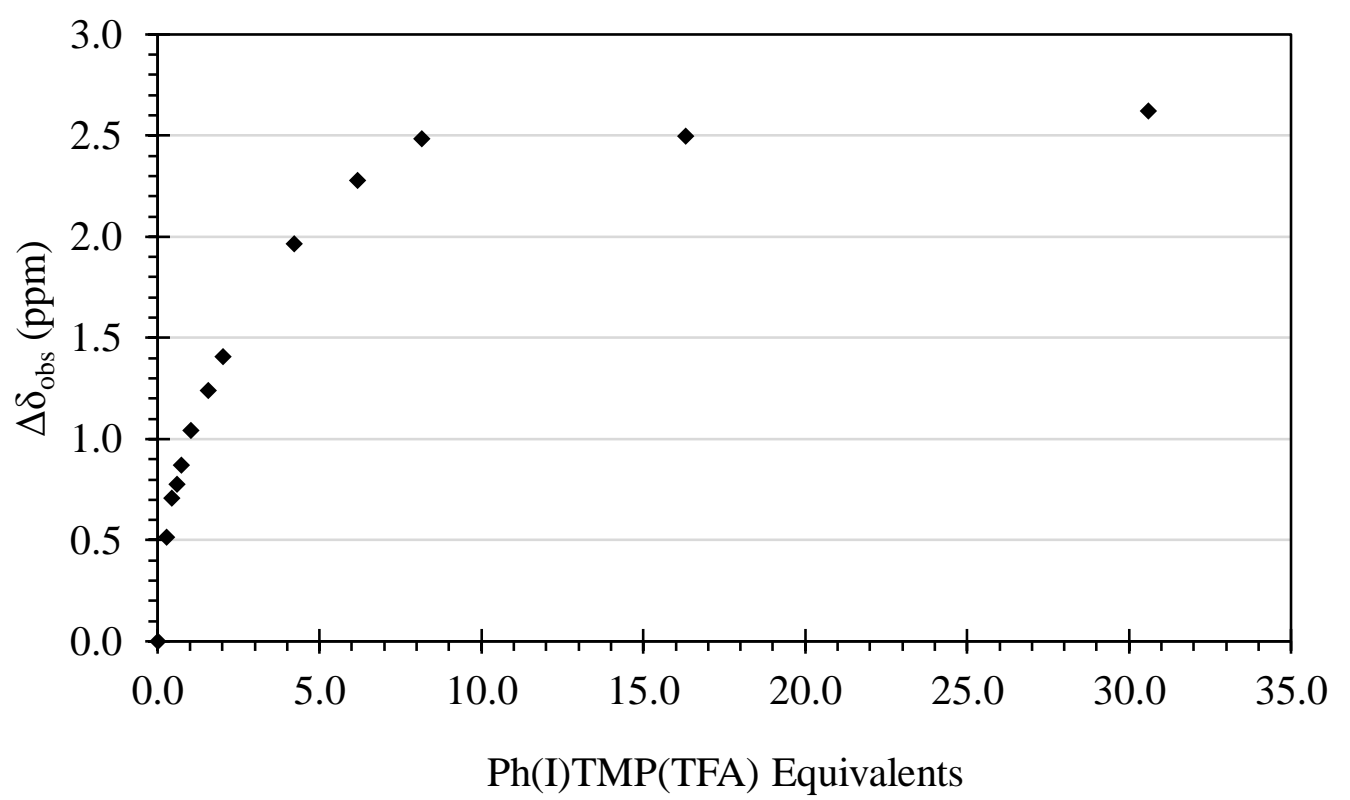

Figure 12. Experimental titration curve for $\mathrm{Ph}(\mathrm{I}) \mathrm{TMP}(\mathrm{TFA})$ in $\mathrm{CD}_{3} \mathrm{CN}$

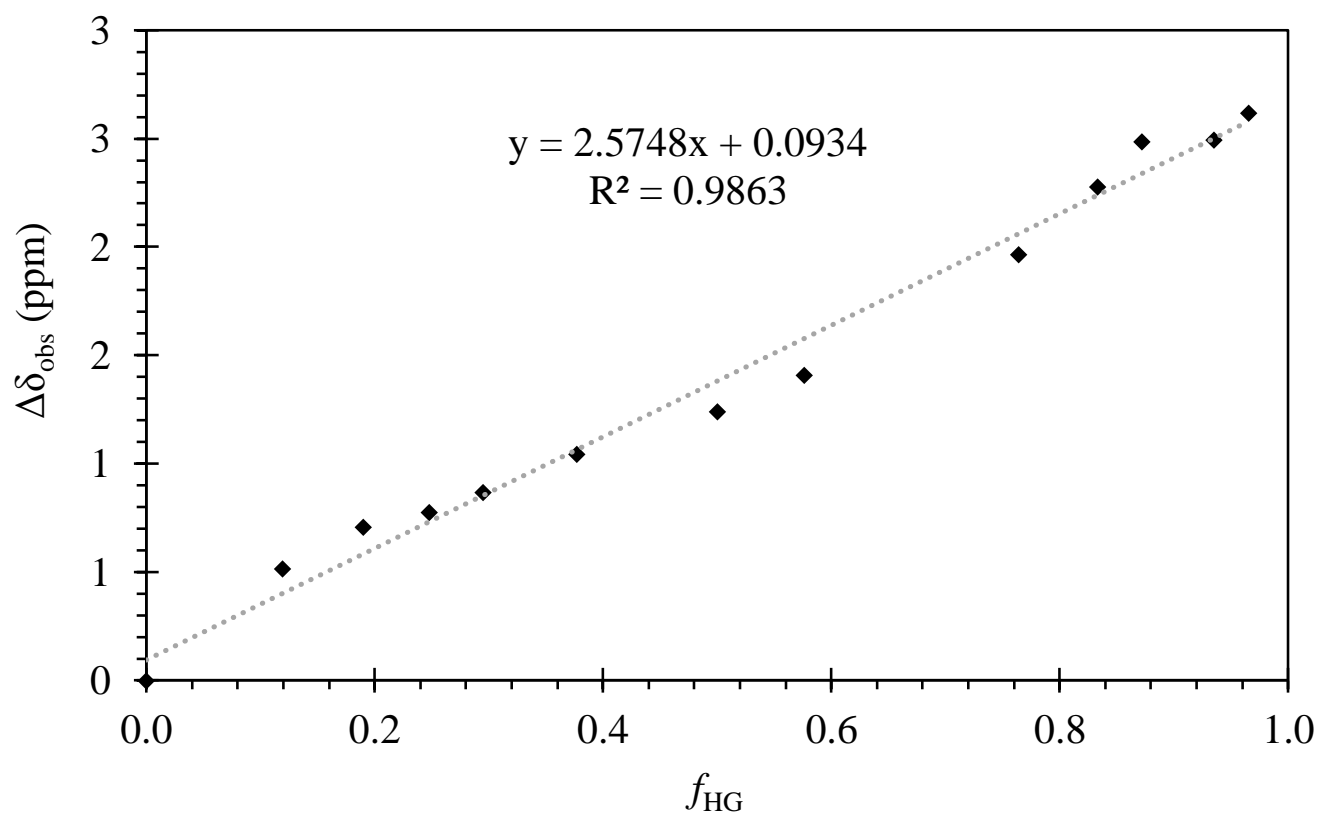

Figure 13. Linear plot for $\mathrm{Ph}(\mathrm{I}) \mathrm{TMP}(\mathrm{TFA})$ in $\mathrm{CD}_{3} \mathrm{CN}$

\subsection{Phenyl(TMP)Iodonium Triflate}

The OTf salt (1d) is shown below in Figure 14 and Figure 15. This salt was also studied in deuterated acetonitrile, with a TPO concentration of $21.21 \mathrm{mM}$. The association constant was determined to be $K_{a}=25.61 \mathrm{M}-1$ and $\Delta \delta_{\max }=5.9234 \mathrm{ppm}$. 


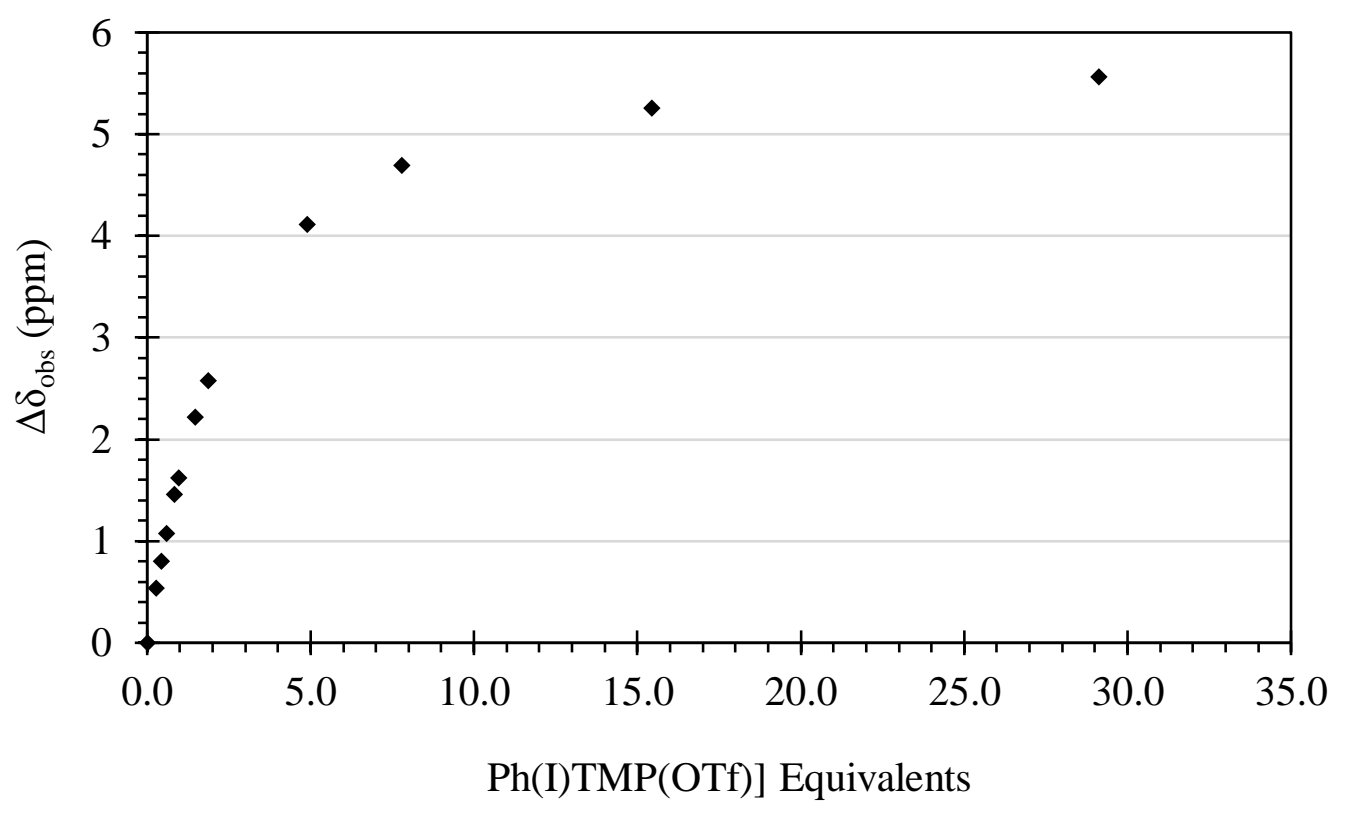

Figure 14. Experimental titration curve for $\operatorname{Ph}(\mathrm{I}) \mathrm{TMP}(\mathrm{OTf})$ in $\mathrm{CD}_{3} \mathrm{CN}$

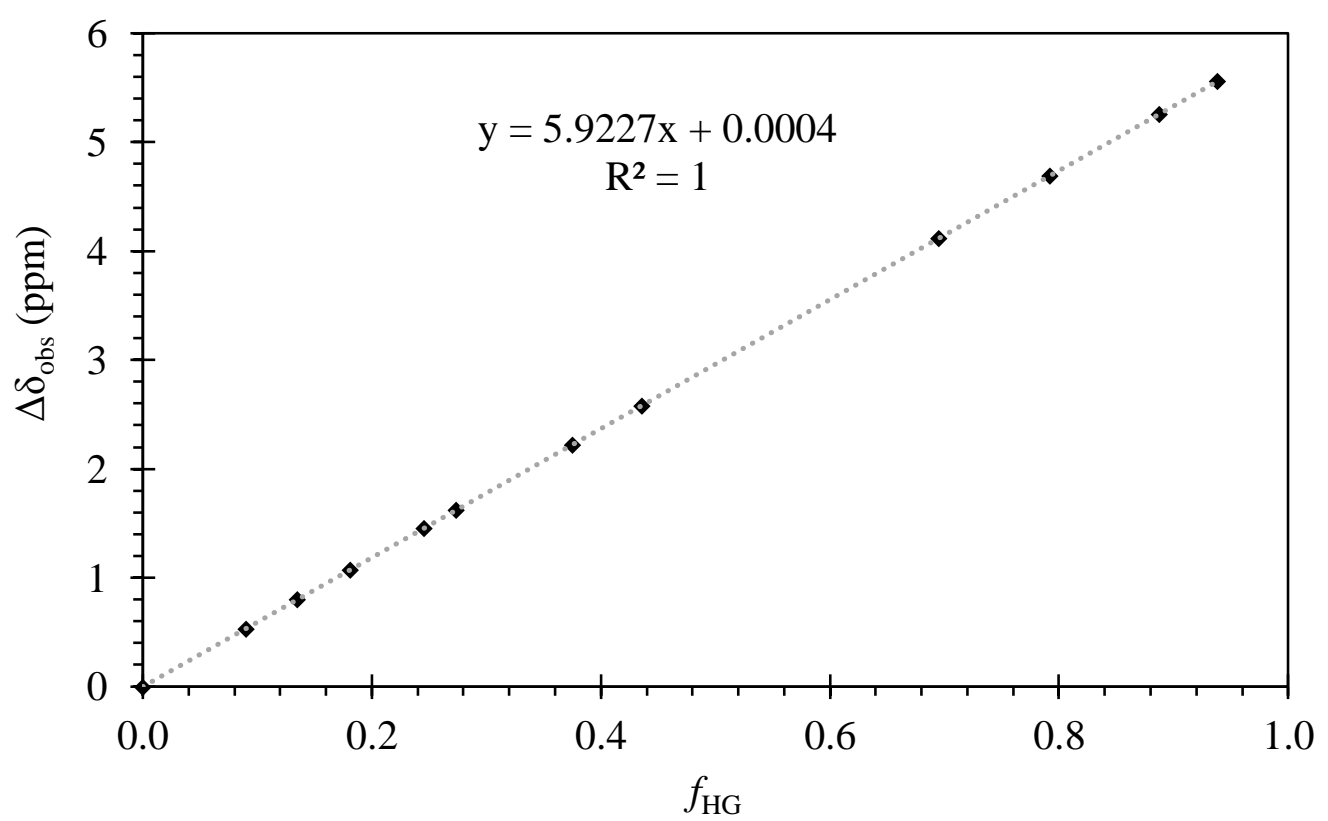

Figure 15. Linear plot for $\mathrm{Ph}(\mathrm{I}) \mathrm{TMP}(\mathrm{OTf})$ in $\mathrm{CD}_{3} \mathrm{CN}$

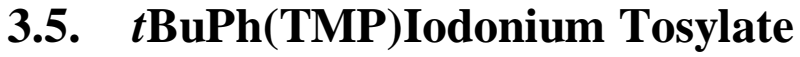

The OTs salt (1e) studied is shown below in Figure 16 and Figure 17. This salt was also studied in deuterated acetonitrile. However, the TPO concentration had to be reduced to $6.69 \mathrm{mM}$ in order to accommodate for the lower solubility of $\mathrm{tBuPh}(\mathrm{TMP}) \mathrm{I}(\mathrm{OTs})$ in $\mathrm{CD}_{3} \mathrm{CN}$. The 
association constant was determined to be $K_{a}=70.42 \mathrm{M}^{-1}$ and $\Delta \delta_{\max }$ was extrapolated to be at $2.3836 \mathrm{ppm}$. However, note that some variation in the experimental procedure yielded a few abnormal data points in the 0 to 2 equivalents range. This variation could be explained by a number of factors including error in volume or mass measurements, mix-up of samples, evaporation of solvent, phosphoric acid capillary leak or other external contamination of sample.

As some of these abnormal data points are above and below the linear regression line (Figure 17), most of the variations balance one another and the fit was deemed adequate for comparison purposes. However, similarly to the TFA salt studied, the extrapolated $K_{a}$ value obtained is disproportionately large. Once again, a very low $\Delta \delta_{\max }$ was recorded, indicating a low Lewis acidity of tBuphenyl(TMP)iodonium tosylate. A $K_{a}$ value of $70.42 \mathrm{M}^{-1}$ is thus clearly inconsistent and not representative of the relative Lewis acidity of this salt. Based on the $\Delta \delta_{\max }$ measured, this tosylate compound has the lowest Lewis acidity of all salts studied.

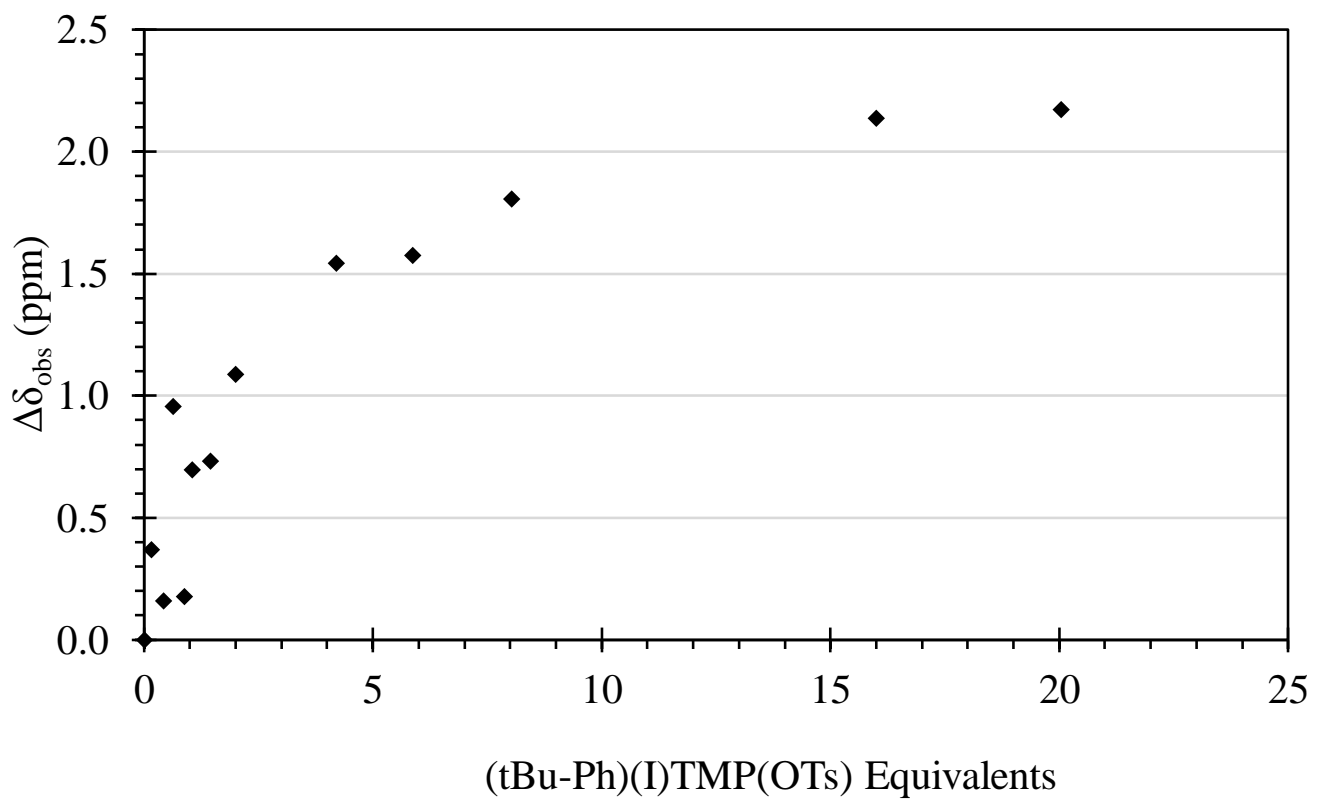

Figure 16. Experimental titration curve for $t \mathrm{BuPh}(\mathrm{I}) \mathrm{TMP}(\mathrm{OTs})$ in $\mathrm{CD}_{3} \mathrm{CN}$ 


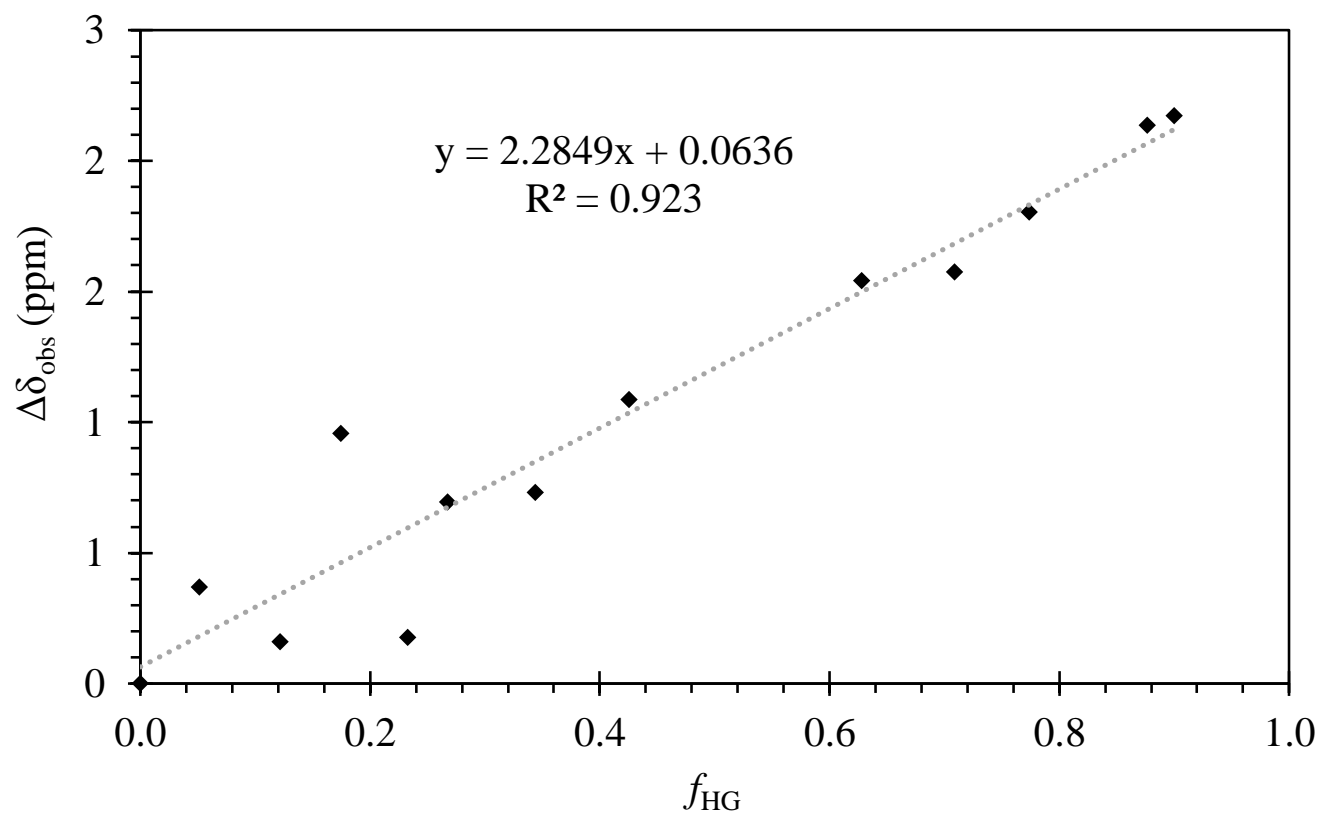

Figure 17. Linear plot for $t \mathrm{BuPh}(\mathrm{I}) \mathrm{TMP}(\mathrm{OTs})$ in $\mathrm{CD}_{3} \mathrm{CN}$

\subsection{Data summary}

Lastly, the juxtaposition of the above experimental titration curves yields Figure 18, which allows for an easier comparison of phenyl(TMP)iodonium salt bearing different counter anions. Clearly, counter anion identity greatly impacts the $\Delta \delta_{\max }$ as will be discussed next. 


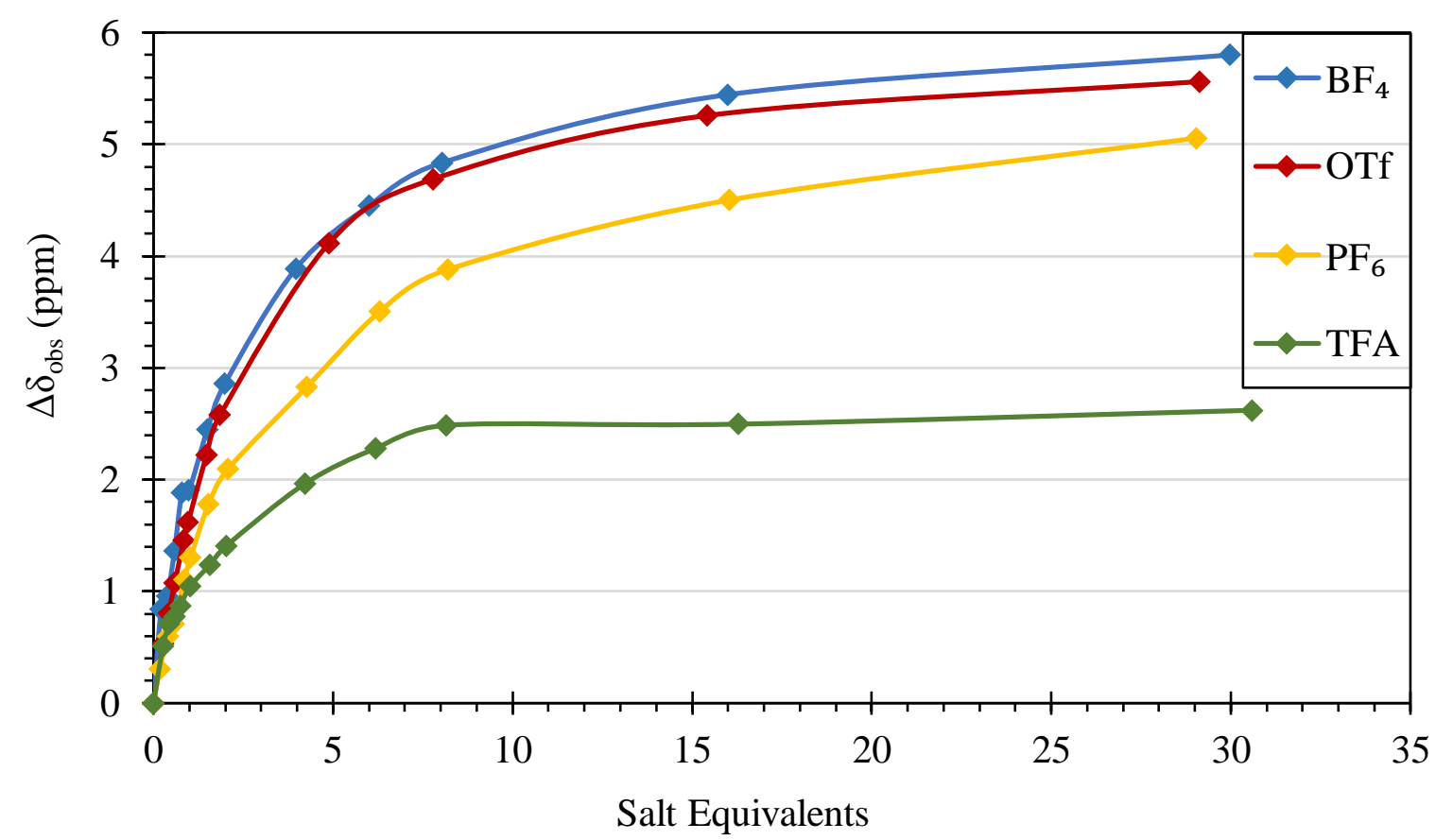

Figure 18. Summary of titration curves for different counter anions

If the collected data for para-tBuphenyl(TMP)iodonium tosylate was added to Figure 18, its curve would be below TFA due to its lower $\Delta \delta_{\max }$ value near $2.3836 \mathrm{ppm}$.

Thanks to the numerical analysis ran on collected data, each of the diaryliodonium salts were ranked from lowest to highest Lewis acidity based on the extrapolated $\Delta \delta_{\max }$ and $K_{a}$ values. Figure 19 below summarizes these findings.

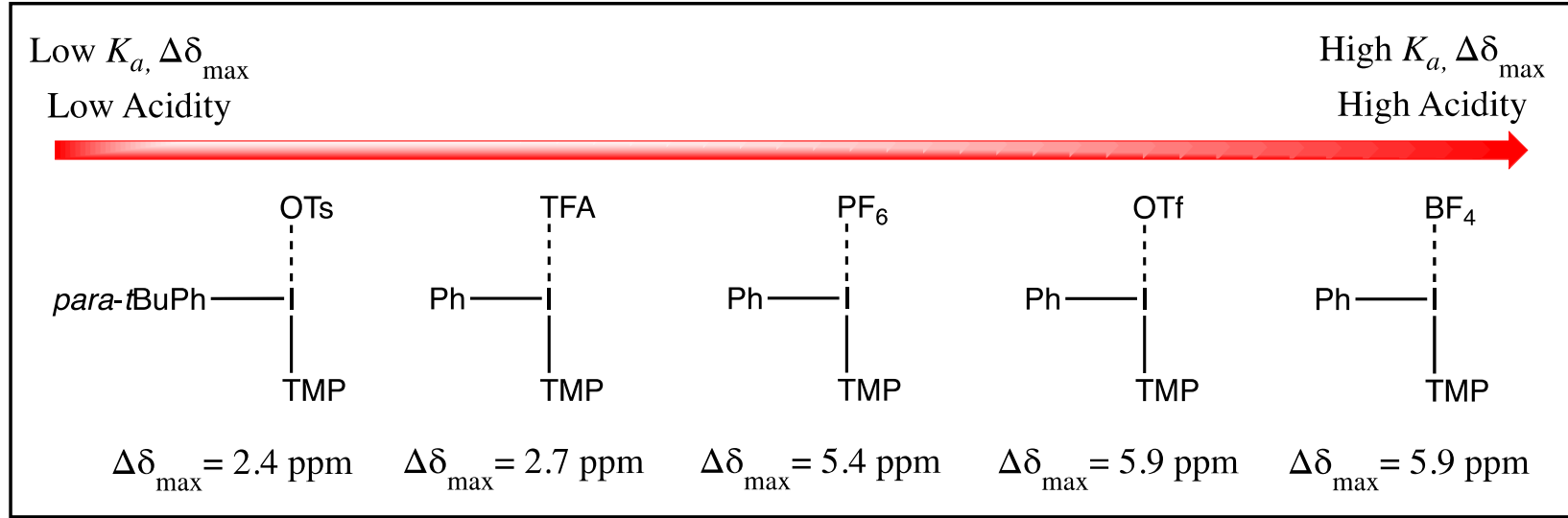

Figure 19. Lewis acidity ranking of diaryliodonium salts 


\section{Discussion}

\subsection{Interpreting the association constant}

Before further analysis of the results, it is important to discuss what lies within the experimental $K_{a}$ value measured. This association constant, or binding constant, is a special case of the equilibrium constant $K$. The equilibrium constant is generally defined as the ratio of the concentrations of the products over reactants, where each component is raised to the power of their stoichiometric coefficients. For example, for a reversible reaction $\mathrm{aA}+\mathrm{bB} \rightarrow \mathrm{cC}+\mathrm{dD}$, the equilibrium constant is defined as $K=\frac{[\mathrm{C}]^{\mathrm{c}}[\mathrm{D}]^{\mathrm{d}}}{[\mathrm{A}]^{\mathrm{a}}[\mathrm{B}]^{\mathrm{b}}}$. On the other hand, the association constant refers more specifically to the binding and unbinding reaction of a host and a guest species, or in our case, of a Lewis acid and base. This reaction can be symbolized as $\mathrm{H}+\mathrm{G} \underset{k_{2}}{\stackrel{k_{1}}{\rightleftharpoons}} \mathrm{HG}$, where $k_{1}$ represents the rate of binding and $k_{2}$ the rate of unbinding. The association constant is then defined

as $K_{a}=\frac{k_{1}}{k_{2}}$. It can also be thought of as the inverse of the dissociation constant, that is $K_{a}=\frac{1}{K_{d}}=$ $\frac{[\mathrm{HG}]}{[\mathrm{H}][\mathrm{G}]}$.

Each Lewis acid (or base), has its own unique association constant. The larger the association constant of an acid, the more associated and electrophilic it is. Thus, the strength of a Lewis acid is measured by the magnitude of its association constant, where a high $K_{a}$ correlates with a high acidity.

\subsection{Observed trend in aryl(TMP)iodonium salts}

First, counter anion effect can be observed within the phenyl(TMP)iodonium salt family. Of these salts, the trifluoroacetate compound revealed to have the lowest Lewis acidity. As a structural analog of acetic acid, TFA is more polar and is thus more likely to compete against TPO 
by complexing to the iodonium center. This competition reduces the effect measured on the base, yielding smaller chemical shift displacements (as seen in Appendix III - TFA) and a smaller $\Delta \delta_{\text {max }}$. On the other hand, the non-coordinating nature of $\mathrm{PF}_{6}$ essentially leaves the iodonium center naked and vulnerable to TPO, resulting in a higher Lewis acidity measured. ${ }^{27}$

Next, Lewis acidity was characterized to be steadily increasing as anions were changed between triflate and tetrafluoroborate respectively. While $\Delta \delta_{\max }$ values recorded were very similar, extrapolated $K_{a}$ values showed the higher acidity of the tetrafluoroborate salt over triflate. Steric effects could also play a part in this interaction between iodonium salts and TPO, making predictions of Lewis acidity measurements more subjective and uncertain.

In addition to counter anion identity, the substitution of diaryliodonium salts can also have important consequences on their Lewis acidity. In their work, Labattut et al. studied three $\mathrm{PF}_{6-}$ bearing salts with electron-withdrawing groups and observed a steady increase in acidity with stronger groups. ${ }^{27}$ The $\Delta \delta_{\max }$ values obtained by Labattut at al. in $\mathrm{CD}_{3} \mathrm{CN}$ increased up to 11.7 ppm, almost twice as much as was observed in aryl(TMP)iodonium salts here. Further studies are needed to explore to Lewis acidity sensitivity of salts bearing electron-donating groups in order to enhance rational selection of these reaction components.

Lastly, it is essential to place these values back in perspective by looking at a few common Lewis acids and how they compare to diaryliodonium salts. For example, $\mathrm{TiCl}_{4}$ and $\mathrm{BF}_{3} \cdot \mathrm{OEt}_{2}$ were previously measured by Labattut et al. in deuterated dichloromethane $\left(\mathrm{CD}_{2} \mathrm{Cl}_{2}\right)$ and thiourea was measured by Hilt et al. using tributylphosphine oxide as a Lewis base in $\mathrm{CD}_{2} \mathrm{Cl}_{2} \cdot{ }^{27,33}$ Although these acids were studied in a different solvent, only marginal decrease in binding affinity was observed by Labattut et al. when switching from $\mathrm{CD}_{2} \mathrm{Cl}_{2}$ to $\mathrm{CD}_{3} \mathrm{CN} .{ }^{27}$ Thus, the fact that $\mathrm{CD}_{3} \mathrm{CN}$ is a slightly more polar and complexing solvent did not greatly affect measurements. 
Respectively, $\Delta \delta_{\max }$ values for TiCl4, BF3 - OEt2 and thiourea were measured to be 44.2 ppm, $28.0 \mathrm{ppm}$ and $7.3 \mathrm{ppm}$. As stated previously, diaryliodonium salts with heavily electronwithdrawing groups displayed a $\Delta \delta_{\max }$ near $11.7 \mathrm{ppm}$ only. Hence, the $\Delta \delta_{\max }$ values obtained in this study, in comparison to these widely different Lewis acids, reveals the relatively mild acidity of aryl(TMP)iodonium salts. 


\section{Conclusion}

By applying the Gutmann-Beckett method to unsymmetrical diaryliodonium salts, it was determined that Lewis acidity increases steadily as counter anions are changed to TFA $\left(\Delta \delta_{\max }=\right.$ $2.7 \mathrm{ppm}), \mathrm{PF}_{6}\left(\Delta \delta_{\max }=5.4 \mathrm{ppm}\right), \mathrm{OTf}\left(\Delta \delta_{\max }=5.9 \mathrm{ppm}\right)$ and $\mathrm{BF}_{4}\left(\Delta \delta_{\max }=5.9 \mathrm{ppm}\right)$ within phenyl(TMP)iodonium salts. The lowest Lewis acidity was recorded in para$t$ Buphenyl(TMP)iodonium tosylate $\left(\Delta \delta_{\max }=2.4 \mathrm{ppm}\right)$.

In comparison to other common Lewis acids, aryl(TMP)iodonium salts display relatively mild Lewis acidity. However, diaryliodonium salts have the advantage of being highly customizable as various aryl groups or counter anions can easily be selected during synthesis as needed. Hence, fine-tuning in Lewis acidity can readily be accomplished by swapping counter anions (oftentimes a one step process), or by altering the electron density of ligands. This high flexibility and reactivity can reveal itself to be invaluable in the synthesis of challenging drugs or agrochemicals.

While these reagents can clearly be exploited as Lewis acids, gaining a better understanding of this unexplored property will hopefully open new doors for synthetic pathways. Perhaps combining the essential arylation reactions that diaryliodonium salts are currently widely being used for with acid-base chemistry will allow new pharmaceutical and agrochemical compounds to be easily synthesized, facilitating mass production and reducing production costs.

Future studies are needed to efficiently utilize diaryliodonium salts as Lewis acids in metalfree arylation reactions. In particular, additional data concerning the effects of electronwithdrawing and electron-donating substituents on Lewis acidity is needed to better predict the uses of diaryliodonium salts. Efficient counter anion screening mechanisms, failing complete understanding of counter anion effect, will also be necessary in order to fully exploit the finetuning aspect of diaryliodonium salts. 


\section{References}

(1) Hartmann, C.; Meyer, V. Ueber eine neue Klasse jodhaltiger, stickstofffreier organischer Basen. Berichte Dtsch. Chem. Ges. 1894, 27 (1), 426-432.

(2) Merritt, E.; Olofsson, B. Diaryliodonium Salts: A Journey from Obscurity to Fame. Angew. Chem. Int. Ed. 2009, 48 (48), 9052-9070.

(3) Based on an April 11, 2018 SciFinder ${ }^{\circledR}$ Search for "Diaryliodonium".

(4) Yoshimura, A.; Zhdankin, V. V. Advances in Synthetic Applications of Hypervalent Iodine Compounds. Chem. Rev. 2016, 116 (5), 3328-3435.

(5) Hypervalent Iodine Chemistry: Modern Developments in Organic Synthesis; Wirth, T., Kita, Y., Eds.; Topics in current chemistry; Springer: Berlin, 2003.

(6) Powell, W. H. Treatment of Variable Valence in Organic Nomenclature (Lambda Convention) (Recommendations 1983). Pure Appl. Chem. 1984, 56 (6), 769-778.

(7) Gillespie, R. The Octet Rule and Hypervalence: Two Misunderstood Concepts. Coord. Chem. Rev. 2002, 233-234, 53-62.

(8) Stirling, A. Assessing Hypervalency in Iodanes. Chem. - Eur. J. 2018, 24 (7), 1709-1713.

(9) Aradi, K.; Tóth, B. L.; Tolnai, G. L.; Novák, Z. Diaryliodonium Salts in Organic Syntheses: A Useful Compound Class for Novel Arylation Strategies. Synlett 2016, 27 (10), 14561485.

(10) Okuyama, T.; Takino, T.; Sueda, T.; Ochiai, M. Solvolysis of Cyclohexenyliodonium Salt, a New Precursor for the Vinyl Cation: Remarkable Nucleofugality of the Phenyliodonio Group and Evidence for Internal Return from an Intimate Ion-Molecule Pair. J. Am. Chem. Soc. 1995, 117 (12), 3360-3367.

(11) Cavallo, G.; Murray, J. S.; Politzer, P.; Pilati, T.; Ursini, M.; Resnati, G. CCDC 1532403: Experimental Crystal Structure Determination. Cambridge Crystallographic Data Centre 2017.

(12) Ledwith, A.; Al-Kass, S.; Sherrington, D. C.; Bonner, P. Ion Pair Dissociation Equilibria for Iodonium and Sulphonium Salts Useful in Photoinitiated Cationic Polymerization. Polymer 1981, 22 (2), 143-145.

(13) Olofsson, B. Arylation with Diaryliodonium Salts. In Hypervalent Iodine Chemistry; Topics in Current Chemistry; Springer, Cham, 2015; pp 135-166.

(14) Seidl, T. L.; Sundalam, S. K.; McCullough, B.; Stuart, D. R. Unsymmetrical Aryl(2,4,6Trimethoxyphenyl)Iodonium Salts: One-Pot Synthesis, Scope, Stability, and Synthetic Studies. J. Org. Chem. 2016, 81 (5), 1998-2009.

(15) Lewis, G. N. Valence and The Structure of Atoms and Molecules; The Chemical Catalog Company, Inc., 1923.

(16) Stuart, D. R. Aryl Transfer Selectivity in Metal-Free Reactions of Unsymmetrical Diaryliodonium Salts. Chem. - Eur. J. 2017.

(17) Seidl, T. The Preparation of Diaryliodonium Salts for Application in Arylation Chemistry; 2018.

(18) Taylor, R. D.; MacCoss, M.; Lawson, A. D. G. Rings in Drugs: Miniperspective. J. Med. Chem. 2014, 57 (14), 5845-5859.

(19) Carey, J. S.; Laffan, D.; Thomson, C.; Williams, M. T. Analysis of the Reactions Used for the Preparation of Drug Candidate Molecules. Org. Biomol. Chem. 2006, 4 (12), 2337. 
(20) Gandy, M. N.; Corral, M. G.; Mylne, J. S.; Stubbs, K. A. An Interactive Database to Explore Herbicide Physicochemical Properties. Org. Biomol. Chem. 2015, 13 (20), 55865590.

(21) Smith, M.; March, J. March's Advanced Organic Chemistry: Reactions, Mechanisms, and Structure, 6th ed.; Wiley-Interscience: Hoboken, N.J, 2007.

(22) Suzuki, A. Cross-Coupling Reactions of Organoboranes: An Easy Way to Construct C-C Bonds (Nobel Lecture). Angew. Chem. Int. Ed Engl. 2011, 50 (30), 6722-6737.

(23) Yusubov, M. S.; Zhdankin, V. V. Iodine Catalysis: A Green Alternative to Transition Metals in Organic Chemistry and Technology. Resour.-Effic. Technol. 2015, 1 (1), 49-67.

(24) Zhang, Y.; Han, J.; Liu, Z.-J. Diaryliodonium Salts as Efficient Lewis Acid Catalysts for Direct Three Component Mannich Reactions. RSC Adv 2015, 5 (32), 25485-25488.

(25) Basdevant, B.; Legault, C. Y. Enantioselective Iodine(III)-Mediated Synthesis of $\alpha$ Tosyloxy Ketones: Breaking the Selectivity Barrier. Org. Lett. 2015, 17 (19), 4918-4921.

(26) Sandtorv, A. H.; Stuart, D. R. Metal-Free Synthesis of Aryl Amines: Beyond Nucleophilic Aromatic Substitution. Angew. Chem. Int. Ed. 2016, 55 (51), 15812-15815.

(27) Labattut, A.; Tremblay, P.-L.; Moutounet, O.; Legault, C. Y. Experimental and Theoretical Quantification of the Lewis Acidity of Iodine(III) Species. J. Org. Chem. 2017.

(28) Mayer, U.; Gutmann, V.; Gerger, W. The Acceptor Number - A Quantitative Empirical Parameter for the Electrophilic Properties of Solvents. Monatshefte Für Chem. 1975, 106 (6), 1235-1257.

(29) Beckett, M. A.; Strickland, G. C.; Holland, J. R.; Sukumar Varma, K. A Convenient NMR Method for the Measurement of Lewis Acidity at Boron Centres: Correlation of Reaction Rates of Lewis Acid Initiated Epoxide Polymerizations with Lewis Acidity. Polymer 1996, 37 (20), 4629-4631.

(30) Benesi, H. A.; Hildebrand, J. H. A Spectrophotometric Investigation of the Interaction of Iodine with Aromatic Hydrocarbons. J. Am. Chem. Soc. 1949, 71 (8), 2703-2707.

(31) Lineweaver, H.; Burk, D. The Determination of Enzyme Dissociation Constants. J. Am. Chem. Soc. 1934, 56 (3), 658-666.

(32) Thordarson, P. Determining Association Constants from Titration Experiments in Supramolecular Chemistry. Chem. Soc. Rev. 2011, 40 (3), 1305-1323.

(33) Nödling, A. R.; Jakab, G.; Schreiner, P. R.; Hilt, G. ${ }^{31}$ P NMR Spectroscopically Quantified Hydrogen-Bonding Strength of Thioureas and Their Catalytic Activity in Diels-Alder Reactions: Hydrogen-Bonding Strength of Thioureas. Eur. J. Org. Chem. 2014, 2014 (29), 6394-6398. 


\section{Appendices}

Appendix I - PF 6

Table 1. Numerical data for PF6 titration curve in $\mathrm{CD}_{3} \mathrm{CN}([\mathrm{TPO}]=20.64 \mathrm{mM})$

\begin{tabular}{ccc}
\hline $\mathrm{PF}_{6}($ equiv $)$ & $\delta_{\text {obs }}(\mathrm{ppm})$ & $\Delta \delta_{\text {obs }}(\mathrm{ppm})$ \\
\hline 0.00 & 51.7370 & 0.00 \\
0.17 & 51.9712 & 0.31 \\
0.41 & 52.2665 & 0.60 \\
0.56 & 52.4491 & 0.71 \\
0.86 & 52.7675 & 1.10 \\
1.03 & 52.9361 & 1.30 \\
1.53 & 53.3699 & 1.78 \\
2.08 & 53.7634 & 2.10 \\
4.27 & 54.7943 & 2.83 \\
6.30 & 55.3291 & 3.50 \\
8.20 & 55.6507 & 3.88 \\
16.05 & 56.2882 & 4.50 \\
29.03 & 56.6428 & 5.06 \\
\hline
\end{tabular}

Numerical fitting was performed using Microsoft ${ }^{\circledR}$ Excel 2018's solver, yielding values of $K_{a}=$ $17.06 \mathrm{M}^{-1}$ and $\Delta \delta_{\max }=5.4012 \mathrm{ppm}$.

\section{Appendix II - $\mathrm{BF}_{4}$}

Table 2. Numerical data for BF4 titration curve in $\mathrm{CD}_{3} \mathrm{CN}([\mathrm{TPO}]=20.64 \mathrm{mM})$

\begin{tabular}{ccc}
\hline $\mathrm{BF}_{4}($ equiv $)$ & $\delta_{\text {obs }}(\mathrm{ppm})$ & $\Delta \delta_{\text {obs }}(\mathrm{ppm})$ \\
\hline 0.00 & 51.2942 & 0.00 \\
0.21 & 52.1381 & 0.84 \\
0.39 & 52.2510 & 0.96 \\
0.58 & 52.6562 & 1.36 \\
0.79 & 53.1757 & 1.88 \\
0.99 & 53.1969 & 1.90 \\
1.50 & 53.7409 & 2.45 \\
1.99 & 54.1565 & 2.86 \\
3.97 & 55.1836 & 3.89 \\
6.01 & 55.7468 & 4.45 \\
8.04 & 56.1305 & 4.84 \\
15.99 & 56.7380 & 5.44 \\
29.99 & 57.0943 & 5.80 \\
\hline
\end{tabular}


Numerical fitting was performed using Microsoft ${ }^{\circledR}$ Excel 2018's solver, yielding values of $K_{a}=$ $32.99 \mathrm{M}^{-1}$ and $\Delta \delta_{\max }=5.9092 \mathrm{ppm}$.

\section{Appendix III - TFA}

Table 3. Numerical data for TFA titration curve in $\mathrm{CD}_{3} \mathrm{CN}([\mathrm{TPO}]=20.16 \mathrm{mM})$

\begin{tabular}{ccc}
\hline TFA (equiv) & $\delta_{\text {obs }}(\mathrm{ppm})$ & $\Delta \delta_{\text {obs }}(\mathrm{ppm})$ \\
\hline 0.00 & 51.2848 & 0.00 \\
0.26 & 51.7988 & 0.51 \\
0.44 & 51.9928 & 0.71 \\
0.60 & 52.0613 & 0.78 \\
0.74 & 52.1545 & 0.87 \\
1.02 & 52.3294 & 1.04 \\
1.57 & 52.5238 & 1.24 \\
2.03 & 52.6930 & 1.41 \\
4.22 & 53.2490 & 1.96 \\
6.18 & 53.5633 & 2.28 \\
8.16 & 53.7708 & 2.49 \\
16.29 & 53.7812 & 2.50 \\
30.59 & 53.9053 & 2.62 \\
\hline
\end{tabular}

Numerical fitting was performed using Microsoft ${ }^{\circledR}$ Excel 2018's solver, yielding values of $K_{a}=$ $46.43 \mathrm{M}^{-1}$ and $\Delta \delta_{\max }=2.7056 \mathrm{ppm}$.

\section{Appendix IV - OTf}

Table 4. Numerical data for OTf titration curve in $\mathrm{CD}_{3} \mathrm{CN}([\mathrm{TPO}]=21.21 \mathrm{mM})$

\begin{tabular}{ccc}
\hline OTf (equiv) & $\delta_{\text {obs }}(\mathrm{ppm})$ & $\Delta \delta_{\text {obs }}(\mathrm{ppm})$ \\
\hline 0.00 & 51.2695 & 0.00 \\
0.27 & 51.8027 & 0.53 \\
0.42 & 52.0707 & 0.80 \\
0.59 & 52.344 & 1.07 \\
0.85 & 52.7259 & 1.46 \\
0.97 & 52.89 & 1.62 \\
1.48 & 53.4913 & 2.22 \\
1.86 & 53.8504 & 2.58 \\
4.89 & 55.3839 & 4.11 \\
7.79 & 55.9586 & 4.69 \\
15.43 & 56.5275 & 5.26 \\
29.13 & 56.8308 & 5.56 \\
\hline
\end{tabular}


Numerical fitting was performed using Microsoft ${ }^{\circledR}$ Excel 2018's solver, yielding values of $K_{a}=$ $25.61 \mathrm{M}^{-1}$ and $\Delta \delta_{\max }=5.9234 \mathrm{ppm}$.

\section{Appendix V-OTs}

Table 5. Numerical data for OTs titration curve in $\mathrm{CD}_{3} \mathrm{CN}([\mathrm{TPO}]=6.69 \mathrm{mM})$

\begin{tabular}{ccc}
\hline OTs (equiv) & $\delta_{\text {obs }}(\mathrm{ppm})$ & $\Delta \delta_{\text {obs }}(\mathrm{ppm})$ \\
\hline 0.00 & 51.9778 & 0.00 \\
0.17 & 52.3472 & 0.37 \\
0.42 & 52.1365 & 0.16 \\
0.62 & 52.9344 & 0.96 \\
0.87 & 52.1546 & 0.18 \\
1.04 & 52.6741 & 0.70 \\
1.46 & 52.7084 & 0.73 \\
2.00 & 53.0656 & 1.09 \\
4.21 & 53.5192 & 1.54 \\
5.87 & 53.5530 & 1.58 \\
8.04 & 53.7818 & 1.80 \\
16.00 & 54.1140 & 2.14 \\
20.04 & 54.1500 & 2.17 \\
\hline
\end{tabular}

Numerical fitting was performed using Microsoft ${ }^{\circledR}$ Excel 2018's solver, yielding values of $K_{a}=$ $70.42 \mathrm{M}^{-1}$ and $\Delta \delta_{\max }=2.3836 \mathrm{ppm}$. 